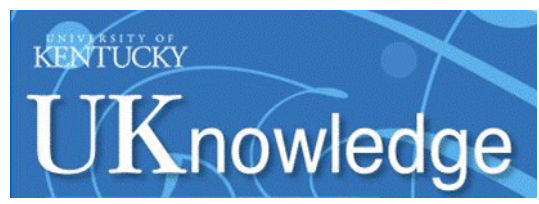

University of Kentucky

UKnowledge

$5-7-2014$

\title{
On the Observability of Optically Thin Coronal Hyperfine Structure Lines
}

\author{
Marios Chatzikos \\ University of Kentucky, mchatzikos@uky.edu
}

Gary J. Ferland

University of Kentucky, gary@uky.edu

R. J. R. Williams

AWE plc, United Kingdom

A. C. Fabian

Institute of Astronomy, United Kingdom

Follow this and additional works at: https://uknowledge.uky.edu/physastron_facpub

Part of the Astrophysics and Astronomy Commons, and the Physics Commons

Right click to open a feedback form in a new tab to let us know how this document benefits you.

\section{Repository Citation}

Chatzikos, Marios; Ferland, Gary J.; Williams, R. J. R.; and Fabian, A. C., "On the Observability of Optically Thin Coronal Hyperfine Structure Lines" (2014). Physics and Astronomy Faculty Publications. 55.

https://uknowledge.uky.edu/physastron_facpub/55

This Article is brought to you for free and open access by the Physics and Astronomy at UKnowledge. It has been accepted for inclusion in Physics and Astronomy Faculty Publications by an authorized administrator of UKnowledge. For more information, please contact UKnowledge@lsv.uky.edu. 
On the Observability of Optically Thin Coronal Hyperfine Structure Lines

Digital Object Identifier (DOI)

https://doi.org/10.1088/0004-637X/787/2/96

Notes/Citation Information

Published in The Astrophysical Journal, v. 787, no. 2, 96, p. 1-15.

(c) 2014. The American Astronomical Society. All rights reserved. Printed in the U.S.A.

The copyright holder has granted permission for posting the article here.

This article is available at UKnowledge: https://uknowledge.uky.edu/physastron_facpub/55 


\title{
ON THE OBSERVABILITY OF OPTICALLY THIN CORONAL HYPERFINE STRUCTURE LINES
}

\author{
M. Chatzikos $^{1}$, G. J. Ferland ${ }^{1}$, R. J. R. Williams ${ }^{2}$, and A. C. Fabian ${ }^{3}$ \\ ${ }^{1}$ University of Kentucky, Lexington, KY 40506, USA; mchatzikos@gmail.com \\ ${ }^{2}$ AWE plc, Aldermaston, Reading RG7 4PR, UK \\ ${ }^{3}$ Institute of Astronomy, Madingley Road, Cambridge CB3 0HA, UK \\ Received 2014 January 14; accepted 2014 April 2; published 2014 May 7
}

\begin{abstract}
We present CLOUdy calculations for the intensity of coronal hyperfine lines in various environments. We model indirect collisional and radiative transitions, and quantify the collisionally excited line emissivity in the density-temperature phase space. As an observational aid, we also express the emissivity in units of that in the 0.4-0.7 keV band. For most hyperfine lines, knowledge of the X-ray surface brightness and the plasma temperature is sufficient for rough estimates. We find that the radiation fields of both Perseus A and Virgo A can enhance the populations of highly ionized species within $1 \mathrm{kpc}$. They can also enhance line emissivity within the cluster core. This could have implications for the interpretation of spectra around bright active galactic nuclei. We find the intensity of the ${ }^{57} \mathrm{Fe}$ XXIV $\lambda 3.068 \mathrm{~mm}$ line to be about two orders of magnitude fainter than previously thought, at $\sim 20 \mu \mathrm{K}$. Comparably bright lines may be found in the infrared. Finally, we find the intensity of hyperfine lines in the Extended Orion Nebula to be low, due to the shallow sightline. Observations of coronal hyperfine lines will likely be feasible with the next generation of radio and submillimeter telescopes.
\end{abstract}

Key words: galaxies: clusters: individual (Perseus, Virgo) - galaxies: individual (NGC 1275, M87) - ISM: individual objects (Extended Orion Nebula) - line: formation - methods: numerical - radio lines: general

Online-only material: color figures, figure sets

\section{INTRODUCTION}

Detections of hyperfine lines may provide vital clues about the astrophysical sources in which they are observed. The most important information that may be gleaned is the abundance of the emitting ion, and, if the line profile is resolved, the magnitude of turbulent motions. Reliable isotopic abundances promise to place tighter constraints on models of primordial and stellar nucleosynthesis, while measuring turbulent motions is of utmost importance for the physics of galaxy clusters (Sunyaev et al. 2003) and molecular clouds. In addition, if the source kinematics are independently known and well understood, the exact line wavelength may provide constraints on models of atomic physics.

Hyperfine transitions take place primarily through the interaction of the magnetic field produced by the electrons and a nucleus with a non-zero magnetic moment. All nuclei possess nonzero magnetic moments, except those with even numbers for both protons and neutrons. The magnetic interaction causes a splitting of energy levels with differentials that typically correspond to radio and millimeter photons. Note that for negative nuclear magnetic moments, the sublevel with the higher angular momentum corresponds to the lower hyperfine level. Due to the low energy differences, excited hyperfine levels have long lifetimes, and vanishingly small transition probabilities. Coupled with the low abundances of these isotopes, hyperfine lines have proven extremely challenging to observe.

To date, only a handful of hyperfine transition lines have been observed astrophysically. The most important is the $21 \mathrm{~cm}$ atomic hydrogen line, which has been observed in the Milky Way (e.g., Dickey \& Lockman 1990), as well as in other galaxies (e.g., Tully \& Fisher 1977). Other lines have been vigorously sought for with varying degrees of success. The $91.6 \mathrm{~cm}$ atomic deuterium line has been observed near the Galactic anti-center (Rogers et al. 2007), while the ${ }^{3} \mathrm{He}$ II $8.665 \mathrm{GHz}$ line has been observed in Galactic planetary nebulae (Bania et al. 2007), as well as in H II regions (Bania et al. 1997). For reference, the observed brightness temperature of the latter is about $1 \mathrm{mK}$, and a detection with the $140 \mathrm{ft}$ Green Bank telescope required a few thousand hours of exposure (Rood et al. 2007).

Despite the observational difficulties, hyperfine transitions have been theoretically studied in several astrophysical contexts. Syunyaev \& Churazov (1984) studied hyperfine lines associated with highly ionized gas, $T>10^{6} \mathrm{~K}$, in supernova remnants and cool core clusters. They determined the primary line excitation mechanism to be resonant scattering through the $2 p$ or $2 s$ levels. They also calculated the properties of lines arising from hydrogenic, lithium-, and sodium-like ions for all elements up to cobalt, which we use extensively in this paper. They predicted that the most important lines should be the hydrogenic ${ }^{14} \mathrm{~N} \lambda 5.6519 \mathrm{~mm}$, and the lithium-like ${ }^{57} \mathrm{Fe} \lambda 3.068 \mathrm{~mm}$ lines. Recently, Sunyaev \& Docenko (2007) made refined predictions for a number of lines emanating from supernovae remnants and other Galactic structures, and obtained best-case brightness temperatures of the order of $100 \mu \mathrm{K}$.

On the other hand, D'Cruz et al. (1998, hereafter DSD98) examined in detail a number of excitation processes for the ${ }^{57} \mathrm{Fe}$ XXIV line, and concluded that electron impact excitation through the first excited level $(2 p)$ is the most important excitation mechanism. They parameterized the emission in the hyperfine line by considering the ratio of its intensity to that of the X-ray doublet at $10.6 \AA$, estimated to about $10^{-7}$. A nearby cool core cluster should yield an antenna temperature of $\sim 0.5 \mathrm{mK}$ if observed with a telescope such as the NRAO $12 \mathrm{~m}$ Telescope (C. L. Sarazin 2013, private communication). Previously, Liang et al. (1997) had unsuccessfully attempted to detect the line in four nearby clusters in their observations with the $22 \mathrm{~m}$ Morpa Telescope.

In this paper, we employ the development version of the spectral synthesis code CLOUdy (Ferland et al. 2013) at 
Table 1

Isotopes of Astrophysical Interest with Finite Magnetic Moment

\begin{tabular}{|c|c|c|c|}
\hline $\begin{array}{l}\text { Isotope } \\
\text { (1) }\end{array}$ & $\begin{array}{l}\text { Spin } \\
(2)\end{array}$ & $\begin{array}{c}\mu_{N}^{\mathrm{a}} \\
(3)\end{array}$ & $\begin{array}{l}\alpha_{\text {iso }}{ }^{b} \\
(4)\end{array}$ \\
\hline${ }^{1} \mathrm{H}$ & 0.5 & 2.792847 & $1.0000 \mathrm{E}+00$ \\
\hline${ }^{2} \mathrm{H}$ & 1.0 & 0.857438 & $1.6500 \mathrm{E}-05$ \\
\hline${ }^{3} \mathrm{He}$ & 0.5 & -2.127498 & $1.4127 \mathrm{E}-05$ \\
\hline${ }^{6} \mathrm{Li}$ & 1.0 & 0.822047 & $8.5009 \mathrm{E}-13$ \\
\hline${ }^{7} \mathrm{Li}$ & 1.5 & 3.256427 & $1.0350 \mathrm{E}-11$ \\
\hline${ }^{9} \mathrm{Be}$ & 1.5 & -1.177432 & $2.4000 \mathrm{E}-11$ \\
\hline${ }^{10} \mathrm{~B}$ & 3.0 & 1.800645 & $9.9701 \mathrm{E}-11$ \\
\hline${ }^{11} \mathrm{~B}$ & 1.5 & 2.688649 & $4.0131 \mathrm{E}-10$ \\
\hline${ }^{13} \mathrm{C}$ & 0.5 & 0.702412 & $8.9668 \mathrm{E}-06$ \\
\hline${ }^{14} \mathrm{~N}$ & 1.0 & 0.403761 & $6.7446 \mathrm{E}-05$ \\
\hline${ }^{15} \mathrm{~N}$ & 0.5 & -0.283189 & $1.5481 \mathrm{E}-07$ \\
\hline${ }^{17} \mathrm{O}$ & 2.5 & -1.893790 & $1.8571 \mathrm{E}-07$ \\
\hline${ }^{19} \mathrm{~F}$ & 0.5 & 2.628868 & $3.6301 \mathrm{E}-08$ \\
\hline${ }^{21} \mathrm{Ne}$ & 1.5 & -0.661797 & $1.8961 \mathrm{E}-07$ \\
\hline${ }^{23} \mathrm{Na}$ & 1.5 & 2.217522 & $1.7400 \mathrm{E}-06$ \\
\hline${ }^{25} \mathrm{Mg}$ & 2.5 & -0.855450 & $3.9801 \mathrm{E}-06$ \\
\hline${ }^{27} \mathrm{Al}$ & 2.5 & 3.641507 & $2.8200 \mathrm{E}-06$ \\
\hline${ }^{29} \mathrm{Si}$ & 0.5 & -0.555290 & $1.5174 \mathrm{E}-06$ \\
\hline${ }^{31} \mathrm{P}$ & 0.5 & 1.131600 & $2.5700 \mathrm{E}-07$ \\
\hline${ }^{33} \mathrm{~S}$ & 1.5 & 0.643821 & $1.0032 \mathrm{E}-07$ \\
\hline${ }^{35} \mathrm{Cl}$ & 1.5 & 0.821874 & $2.3947 \mathrm{E}-07$ \\
\hline${ }^{37} \mathrm{Cl}$ & 1.5 & 0.684124 & $7.6536 \mathrm{E}-08$ \\
\hline${ }^{39} \mathrm{~K}$ & 1.5 & 0.391470 & $9.9653 \mathrm{E}-08$ \\
\hline${ }^{41} \mathrm{~K}$ & 1.5 & 0.214870 & $7.1916 \mathrm{E}-09$ \\
\hline${ }^{43} \mathrm{Ca}$ & 3.5 & -1.317300 & $2.9565 \mathrm{E}-09$ \\
\hline${ }^{45} \mathrm{Sc}$ & 3.5 & 4.756487 & $1.4100 \mathrm{E}-09$ \\
\hline${ }^{47} \mathrm{Ti}$ & 2.5 & -0.788480 & $6.6291 \mathrm{E}-09$ \\
\hline${ }^{49} \mathrm{Ti}$ & 3.5 & -1.104170 & $4.8204 \mathrm{E}-09$ \\
\hline${ }^{51} \mathrm{~V}$ & 3.5 & 5.148706 & $8.4889 \mathrm{E}-09$ \\
\hline${ }^{53} \mathrm{Cr}$ & 1.5 & -0.474540 & $4.1520 \mathrm{E}-08$ \\
\hline${ }^{55} \mathrm{Mn}$ & 2.5 & 3.453200 & $2.6900 \mathrm{E}-07$ \\
\hline${ }^{57} \mathrm{Fe}$ & 0.5 & 0.090764 & $6.6962 \mathrm{E}-07$ \\
\hline${ }^{59} \mathrm{Co}$ & 3.5 & 4.627000 & $9.7702 \mathrm{E}-08$ \\
\hline
\end{tabular}

Notes.

a Nuclear magnetic moment, in units of nuclear magnetons, $\mu_{N}$ (Stone 2005).

b Abundance relative to hydrogen. See the text.

revision r8480 to extend previous calculations. CLOUDY is able to solve for the ionization structure of a plasma, self-consistently accounting for radiative and collisional processes.

Table 1 lists the nuclear properties of all astrophysically important isotopes, as used by the code. Column 1 identifies the isotope, while Columns 2 and 3 list the nuclear spin and the nuclear magnetic moment in units of nuclear magnetons (Stone 2005), respectively. The isotopic abundance relative to hydrogen is listed in Column 4. The solar abundances of Grevesse et al. (2010) and the isotope fractions of Asplund et al. (2009) are employed in this work. The latter are modified so that the deuterium-to-hydrogen ratio is primordial (Pettini \& Bowen 2001 ), and the ${ }^{13} \mathrm{C}$ isotope fraction is $1 / 30$.

Tables 2, 3, and 4 present, respectively, the hydrogenic, lithium-like, and sodium-like coronal lines of Syunyaev \& Churazov (1984). Column 1 identifies the emission line, while Columns 2 and 3 give the Einstein $A$ and the transition wavelength, respectively, as given by the reference in Column 4.

We improve upon previous work by accounting for impact excitations through all levels higher than $2 p$, and by considering the radiative effects due to point source radiation fields. These are discussed in detail in Section 2. In Section 3, we present
Table 2

Hydrogenic Coronal Lines

\begin{tabular}{|c|c|c|c|}
\hline Line & $\begin{array}{c}A_{\mathrm{ul}} \\
\left(\mathrm{s}^{-1}\right) \\
(2)\end{array}$ & $\begin{array}{c}\lambda \\
(\mathrm{cm}) \\
(3)\end{array}$ & Ref. $^{\mathrm{a}}$ \\
\hline${ }^{1} \mathrm{H}$ I & $2.8843 \mathrm{E}-15$ & 21.1207 & 1 \\
\hline${ }^{2} \mathrm{H} \mathrm{I}$ & $4.6968 \mathrm{E}-17$ & 91.6354 & 1 \\
\hline${ }^{3} \mathrm{He}$ II & $1.9544 \mathrm{E}-12$ & 3.46194 & 1 \\
\hline${ }^{6} \mathrm{Li}$ III & $8.1000 \mathrm{E}-13$ & 3.53 & 2 \\
\hline${ }^{7} \mathrm{Li}$ III & $4.0000 \mathrm{E}-11$ & 1 & 2 \\
\hline${ }^{9} \mathrm{Be}$ IV & $4.2000 \mathrm{E}-11$ & 1.17 & 2 \\
\hline${ }^{10} \mathrm{~B} \mathrm{~V}$ & $5.1000 \mathrm{E}-10$ & 0.447 & 2 \\
\hline${ }^{11} \mathrm{~B} \mathrm{~V}$ & $2.2000 \mathrm{E}-09$ & 0.262 & 2 \\
\hline${ }^{13} \mathrm{C}$ VI & $4.6000 \mathrm{E}-10$ & 0.3874 & 3 \\
\hline${ }^{14} \mathrm{~N}$ VII & $2.0000 \mathrm{E}-10$ & 0.56519 & 3 \\
\hline${ }^{15} \mathrm{~N}$ VII & $3.6000 \mathrm{E}-10$ & 0.603 & 2 \\
\hline${ }^{17} \mathrm{O}$ VIII & $6.2000 \mathrm{E}-08$ & 0.10085 & 3 \\
\hline${ }^{19} \mathrm{~F}$ IX & $9.6000 \mathrm{E}-07$ & 0.0305 & 2 \\
\hline${ }^{21} \mathrm{Ne} \mathrm{x}$ & $2.8000 \mathrm{E}-08$ & 0.132 & 2 \\
\hline${ }^{23} \mathrm{Na} \mathrm{XI}$ & $1.6000 \mathrm{E}-06$ & 0.0296 & 2 \\
\hline${ }^{25} \mathrm{Mg}$ XII & $2.2000 \mathrm{E}-07$ & 0.0654 & 2 \\
\hline${ }^{27} \mathrm{Al}$ XIII & $2.5000 \mathrm{E}-05$ & 0.0121 & 2 \\
\hline${ }^{29} \mathrm{Si} \mathrm{XIV}$ & $1.5000 \mathrm{E}-06$ & 0.0379 & 2 \\
\hline${ }^{31} \mathrm{P} \times V$ & $7.8000 \mathrm{E}-06$ & 0.0151 & 2 \\
\hline${ }^{33} \mathrm{~S}$ XVI & $1.2000 \mathrm{E}-06$ & 0.0327 & 2 \\
\hline${ }^{35} \mathrm{Cl}$ XVII & $4.2000 \mathrm{E}-06$ & 0.0213 & 2 \\
\hline${ }^{37} \mathrm{Cl}$ XVII & $2.4000 \mathrm{E}-06$ & 0.0255 & 2 \\
\hline${ }^{39} \mathrm{~K}$ XIX & $1.3000 \mathrm{E}-06$ & 0.0317 & 2 \\
\hline${ }^{41} \mathrm{~K}$ XIX & $2.1000 \mathrm{E}-07$ & 0.0578 & 2 \\
\hline${ }^{43} \mathrm{Ca} \mathrm{XX}$ & $7.3000 \mathrm{E}-05$ & 0.0094 & 2 \\
\hline${ }^{45} \mathrm{Sc}$ XXI & $4.2000 \mathrm{E}-03$ & 0.00224 & 2 \\
\hline${ }^{47}$ Ti XXII & $4.5000 \mathrm{E}-05$ & 0.0111 & 2 \\
\hline${ }^{49} \mathrm{Ti}$ XXII & $1.0000 \mathrm{E}-04$ & 0.00835 & 2 \\
\hline${ }^{51} \mathrm{~V}$ XXIII & $1.2000 \mathrm{E}-02$ & 0.00156 & 2 \\
\hline${ }^{53} \mathrm{Cr}$ XXIV & $3.3000 \mathrm{E}-05$ & 0.0127 & 2 \\
\hline${ }^{55} \mathrm{Mn}$ XXV & $8.9000 \mathrm{E}-05$ & 0.00171 & 2 \\
\hline${ }^{57} \mathrm{Fe}$ XXVI & $6.4000 \mathrm{E}-07$ & 0.0348 & 2 \\
\hline${ }^{59}$ Co XXVII & $4.1000 \mathrm{E}-02$ & 0.00105 & 2 \\
\hline
\end{tabular}

Note. ${ }^{a}$ Wavelength and transition probability reference: (1) Gould (1994); (2) Syunyaev \& Churazov (1984); (3) Shabaev et al. (1995).

grids of calculations for the hyperfine line emissivities on the density-temperature plane. In Section 4, we present calculations for the Perseus and Virgo galaxy clusters, as well as the Extended Orion Nebula, and discuss their implications in Section 5. We summarize in Section 6.

\section{LINE EXCITATION PROCESSES}

\subsection{Collisional Excitations}

\subsubsection{Direct and Resonant Excitations}

Syunyaev \& Churazov (1984) examined a number of line excitation processes and concluded that resonant excitation through the first level above ground is the primary excitation mechanism for the coronal hyperfine lines of Tables 2-4. At the suggestion of DSD98, Zhang \& Sampson (2000, 2001, hereafter ZS00) performed detailed calculations of the collision strength for direct and resonant excitation of hyperfine lines for a number of hydrogenic and lithium-like ions. Their findings suggest that resonant excitations indeed dominate direct excitations at temperatures below $\sim 5 \times 10^{7} \mathrm{~K}$. In general, resonant excitations are more important than direct excitations.

Goddard \& Ferland (2003) provided fits to these collisional data of about $30 \%$ accuracy for the purposes of incorporating 
Table 3

Lithium-Like Coronal Lines

\begin{tabular}{|c|c|c|c|}
\hline Line & $\begin{array}{c}A_{\mathrm{ul}} \\
\left(\mathrm{s}^{-1}\right) \\
(2)\end{array}$ & $\begin{array}{c}\lambda \\
(\mathrm{cm}) \\
(3)\end{array}$ & $\begin{array}{l}\text { Ref. } \\
\text { (4) }\end{array}$ \\
\hline${ }^{6} \mathrm{Li} I$ & $1.5900 \mathrm{E}-17$ & 131.461 & 1 \\
\hline${ }^{7} \mathrm{Li}$ I & $7.7900 \mathrm{E}-16$ & 37.3364 & 1 \\
\hline${ }^{9} \mathrm{Be}$ II & $4.8900 \mathrm{E}-15$ & 23.999 & 1 \\
\hline${ }^{10} \mathrm{~B}$ III & $1.2000 \mathrm{E}-13$ & 7.2 & 2 \\
\hline${ }^{11} \mathrm{~B}$ III & $5.4000 \mathrm{E}-13$ & 4.22 & 2 \\
\hline${ }^{13} \mathrm{C}$ IV & $1.7000 \mathrm{E}-13$ & 5.39 & 2 \\
\hline${ }^{14} \mathrm{~N} v$ & $9.8000 \mathrm{E}-14$ & 7.072 & 3 \\
\hline${ }^{15} \mathrm{~N} \mathrm{v}$ & $1.8000 \mathrm{E}-13$ & 7.65 & 2 \\
\hline${ }^{17} \mathrm{O}$ VI & $3.6000 \mathrm{E}-11$ & 1.19 & 2 \\
\hline${ }^{19} \mathrm{~F}$ VII & $6.6000 \mathrm{E}-10$ & 0.34102 & 3 \\
\hline${ }^{21} \mathrm{Ne}$ VIII & $2.3000 \mathrm{E}-11$ & 1.43 & 2 \\
\hline${ }^{23} \mathrm{Na} I X$ & $1.3000 \mathrm{E}-09$ & 0.30924 & 3 \\
\hline${ }^{25} \mathrm{Mg} \mathrm{X}$ & $2.1000 \mathrm{E}-10$ & 0.6679 & 3 \\
\hline${ }^{27} \mathrm{Al}$ XI & $2.5000 \mathrm{E}-08$ & 0.1206 & 3 \\
\hline${ }^{29}$ Si XII & $1.5000 \mathrm{E}-09$ & 0.3725 & 3 \\
\hline${ }^{31} \mathrm{P}$ XIII & $8.6000 \mathrm{E}-09$ & 0.146 & 2 \\
\hline${ }^{33} \mathrm{~S}$ XIV & $1.3000 \mathrm{E}-09$ & 0.312 & 2 \\
\hline${ }^{35} \mathrm{Cl} \mathrm{XV}$ & $5.0000 \mathrm{E}-09$ & 0.20073 & 3 \\
\hline${ }^{37} \mathrm{Cl} \times v$ & $2.9000 \mathrm{E}-09$ & 0.241 & 2 \\
\hline${ }^{39} \mathrm{~K}$ XVII & $1.6000 \mathrm{E}-09$ & 0.294 & 2 \\
\hline${ }^{41} \mathrm{~K}$ XVII & $2.6000 \mathrm{E}-10$ & 0.535 & 2 \\
\hline${ }^{43} \mathrm{Ca}$ XVIII & $9.4000 \mathrm{E}-08$ & 0.0862 & 2 \\
\hline${ }^{45} \mathrm{Sc}$ XIX & $5.6000 \mathrm{E}-06$ & 0.0203 & 2 \\
\hline${ }^{47} \mathrm{Ti} \mathrm{xx}$ & $6.2000 \mathrm{E}-08$ & 0.101 & 2 \\
\hline${ }^{49} \mathrm{Ti} \mathrm{xx}$ & $1.4000 \mathrm{E}-07$ & 0.0754 & 2 \\
\hline${ }^{51} \mathrm{~V}$ XXI & $1.7000 \mathrm{E}-05$ & 0.014 & 2 \\
\hline${ }^{53} \mathrm{Cr}$ XXII & $4.7000 \mathrm{E}-08$ & 0.113 & 2 \\
\hline${ }^{55} \mathrm{Mn}$ XXIII & $1.3000 \mathrm{E}-05$ & 0.0152 & 2 \\
\hline${ }^{57} \mathrm{Fe}$ XXIV & $9.4000 \mathrm{E}-10$ & 0.3068 & 3 \\
\hline${ }^{59} \mathrm{Co} \mathrm{XXV}$ & $6.1000 \mathrm{E}-05$ & 0.00915 & 2 \\
\hline
\end{tabular}

Note. ${ }^{a}$ Wavelength and transition probability reference: (1) Garstang (1995); (2) Syunyaev \& Churazov (1984); (3) Shabaev et al. (1995).

them into Cloudy. In Appendix A, we present improved fits to the collision strengths of Zhang \& Sampson of accuracy about $15 \%$, which we use throughout our calculations. Note that we adopt the lithium-like fits for the sodium-like collision strengths, due to lack of data for sodium-like ions.

In the following, we will refer to direct and resonant excitations collectively as "direct" excitations.

\subsubsection{Indirect Impact Excitations}

On the other hand, DSD98 showed that the most important excitation mechanism for the lithium-like ${ }^{57} \mathrm{Fe}$ line is indirect impact collisional excitations through the $2 p$ sublevel. DSD98 also considered excitations through higher levels and concluded that the effect was not significant, of the order of $10 \%$.

We have adapted CLOUDY to include excitations through levels higher than $2 p$. These are drawn from the CHIANTI (Dere et al. 1997; Landi et al. 2013), STоuT (Ferland et al., in preparation), and Opacity Project (Seaton 2005) databases. Note that no radiative or collisional data are available for $\mathrm{Cl}^{+14}$. Our results on the lithium-like $\mathrm{Cl}$ xv lines include only direct excitations.

The importance of these improvements for the lithium-like ${ }^{57} \mathrm{Fe}$ line is illustrated in Figure 1. The collision strengths for direct, indirect through the $2 p$ level, and indirect excitations through all higher levels are compared to the results of DSD98 in the left panel. In agreement with these researchers, the inclusion
Table 4

Sodium-Like Coronal Lines

\begin{tabular}{lcll}
\hline \hline Line & $\begin{array}{c}A_{\text {ul }} \\
\left(\mathrm{s}^{-1}\right)\end{array}$ & $\begin{array}{c}\lambda \\
(\mathrm{cm})\end{array}$ & Ref. $^{\text {a }}$ \\
$(1)$ & $(2)$ & $(3)$ & $(4)$ \\
\hline${ }^{23} \mathrm{NaI}$ & $8.3500 \mathrm{E}-15$ & 16.9336 & 1 \\
${ }^{25} \mathrm{Mg}$ II & $1.3400 \mathrm{E}-14$ & 16.771 & 1 \\
${ }^{27} \mathrm{Al}$ III & $5.5000 \mathrm{E}-12$ & 2 & 2 \\
${ }^{29} \mathrm{Si}$ IV & $6.3000 \mathrm{E}-13$ & 5 & 2 \\
${ }^{31} \mathrm{P}$ v & $6.5000 \mathrm{E}-12$ & 1.6 & 2 \\
${ }^{33} \mathrm{~S}$ VI & $1.3000 \mathrm{E}-12$ & 3.1 & 2 \\
${ }^{35} \mathrm{Cl}$ VII & $7.0000 \mathrm{E}-12$ & 1.8 & 2 \\
${ }^{37} \mathrm{Cl}$ VII & $4.4000 \mathrm{E}-12$ & 2.1 & 2 \\
${ }^{39} \mathrm{~K}$ IX & $3.8000 \mathrm{E}-12$ & 2.2 & 2 \\
${ }^{41} \mathrm{~K}$ IX & $6.2000 \mathrm{E}-13$ & 4 & 2 \\
${ }^{43} \mathrm{Ca} \mathrm{X}$ & $2.7000 \mathrm{E}-10$ & 0.61 & 2 \\
${ }^{45} \mathrm{Sc} \mathrm{XI}$ & $1.8000 \mathrm{E}-08$ & 0.14 & 2 \\
${ }^{47} \mathrm{Ti}$ XII & $2.3000 \mathrm{E}-10$ & 0.65 & 2 \\
${ }^{49} \mathrm{Ti}$ XII & $5.2000 \mathrm{E}-10$ & 0.49 & 2 \\
${ }^{51} \mathrm{~V}$ XIII & $7.0000 \mathrm{E}-08$ & 0.087 & 2 \\
${ }^{53} \mathrm{Cr}$ XIV & $2.1000 \mathrm{E}-10$ & 0.68 & 2 \\
${ }^{55} \mathrm{Mn}$ XV & $6.6000 \mathrm{E}-08$ & 0.089 & 2 \\
${ }^{57} \mathrm{Fe}$ XVI & $5.0000 \mathrm{E}-12$ & 1.8 & 2 \\
${ }^{59} \mathrm{Co}$ XVII & $3.5000 \mathrm{E}-07$ & 0.051 & 2 \\
\hline
\end{tabular}

Note. ${ }^{a}$ Wavelength and transition probability reference: (1) Garstang (1995); (2) Syunyaev \& Churazov (1984).

of indirect collisional excitations from higher levels than $2 p$ increases the effective collision strength by a modest amount that approaches $15 \%$ at $10^{8} \mathrm{~K}$. The collision strength due to the $2 p$ indirect collisions is higher than the one reported in Figure 5 of DSD98, probably due to improvements in atomic physics data since that publication. Overall, the updated collision strengths are higher than those reported by DSD98 by $40 \%-75 \%$.

To complete the comparison with the DSD98 findings, the right panel of Figure 1 presents the hyperfine line intensity in units of the $10.6 \AA \mathrm{X}$-ray doublet. The improved atomic physics leads to a boost in the line ratio at lower temperatures. At the temperature of the ionization fraction peak $(\sim 20 \mathrm{MK})$ it is roughly $50 \%$.

The relative importance of direct and indirect excitations varies across elements and isoelectronic sequences. In the simplest case of hydrogenic ions, the energy above ground varies as $Z^{2}$, so that indirect excitations are not particularly important for heavy ions (e.g., Figure 2, left panel). For the lithium-like and sodium-like sequences, the $\mathrm{K}$ and L shells are, respectively, full, the energy separation between the ground and the first excited state decreases, indirect excitations become favorable, and the relevant collision strength attains large values (e.g., Figure 2, right panel).

\subsection{Optical Pumping}

External radiation fields may affect line emissivity. The Cosmic Microwave Background (CMB) plays an important role for most of the lines considered here, and is included in all the models below. After correcting for the CMB continuum, line emissivity is reduced by a factor (see DSD98, Equations (35) and (36)) that depends on the radiation temperature and atomic physics. This correction is implemented in CLOUDY according to Chatzikos et al. (2013).

On the other hand, radiation from a point source that lies away from the sightline can lead to observable line emission enhancements. This may be accomplished by direct or indirect 

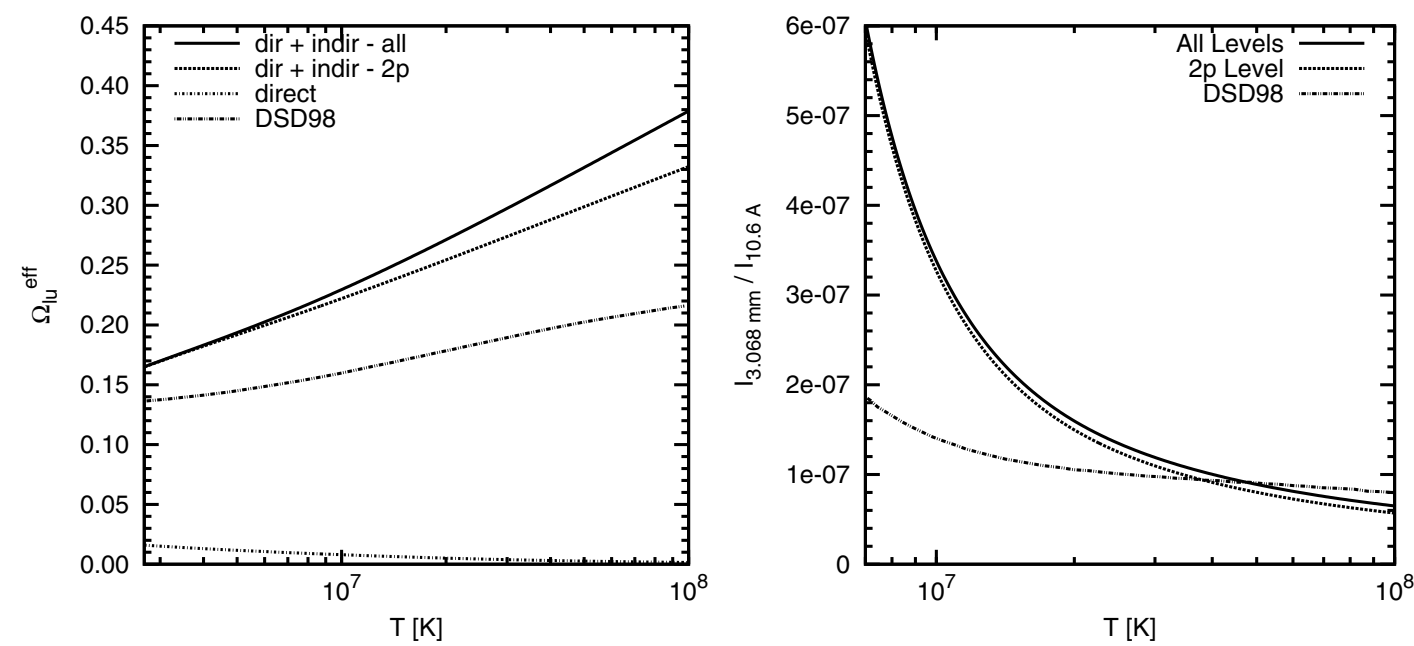

Figure 1. Left panel: effective collision strength as a function of temperature. Right panel: hyperfine line intensity in units of the $10.6 \AA \mathrm{X}$-ray doublet. In both plots, the excitations shown are direct (dot-dashed), indirect through the $2 p$ level (dashed), and through all higher levels in CLoudy (solid). The results of D'Cruz et al. (1998) are also shown (dot-dot-dashed).
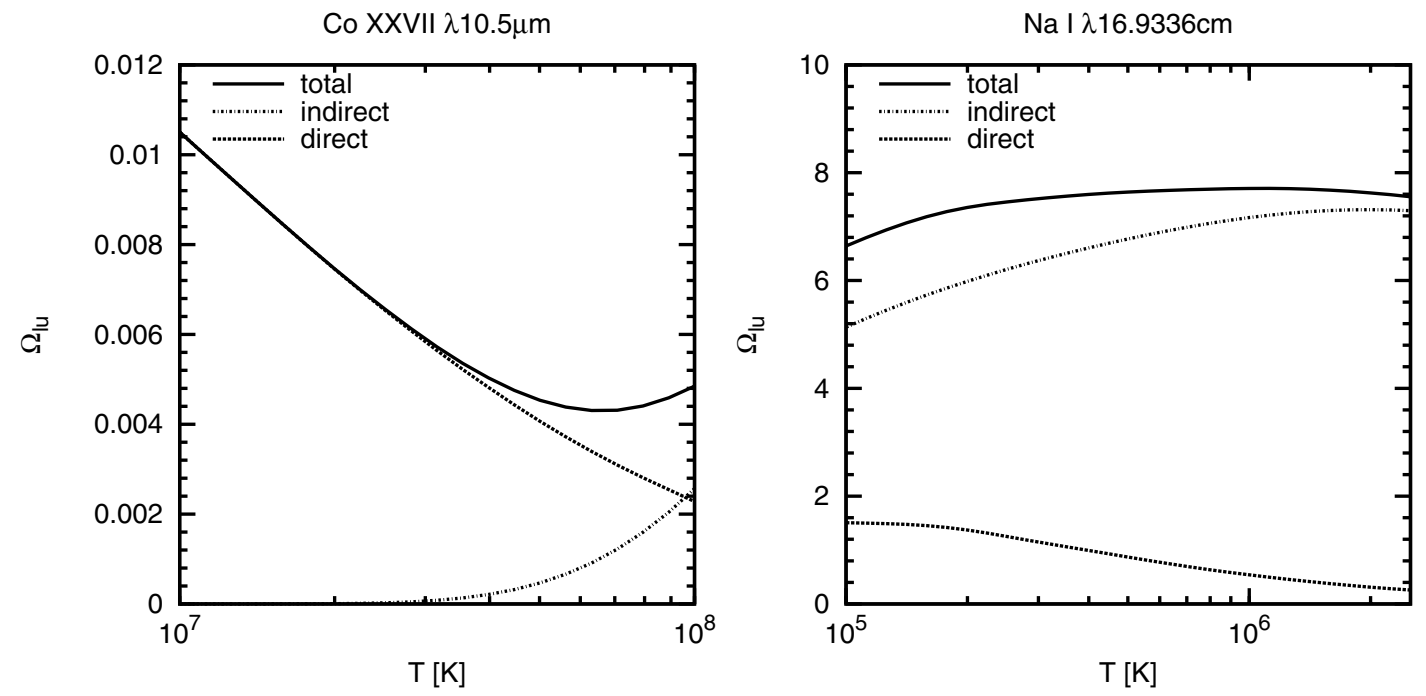

Figure 2. Collision strength decomposition for hydrogenic cobalt (left panel) and neutral sodium (right panel). The dominant mode of collisional excitation depends on the iso-sequence.

radiative excitations that populate the upper hyperfine level. Although the CMB photon occupation number typically exceeds that due to point sources (unless in close proximity to the source), the fact that the radiation does not contribute to continuum translates to a significant boost in the line emissivity. We refer to this process as "optical pumping."

In the case of galaxy clusters, the central active galactic nucleus (AGN) can affect the hyperfine line emissivity and even the ionization structure of the gas. For illustration, we consider the radiation field of NGC 1275 at the center of the Perseus Cluster, although it is an unusually bright source. The compilation of its spectral energy distribution (SED) is presented in Appendix B, where special care is taken to account for frequency-dependent, long-term variability.

The effect of the SED is best demonstrated by examining the properties of the ${ }^{57} \mathrm{Fe}$ xxIV $\lambda 3.068 \mathrm{~mm}$ line as a function of source proximity. The gas density is set to $10^{-3} \mathrm{~cm}^{-3}$ and the volume to $1 \mathrm{~cm}^{3}$.

The left panel in Figure 3 shows the ionization fraction of $\mathrm{Fe}^{+23}$ as a function of temperature and distance to the point source. The SED acts to decrease the maximum ionization fraction for this species and to shift that maximum to lower temperatures. At $5 \mathrm{kpc}$ the maximum fraction and its temperature are reduced by factors of $20 \%$ and 2, respectively, relative to the purely collisional case. Because the number of ionizing photons decreases as $1 / r^{2}, r$ being the distance to the source, these effects are quickly moderated with increasing distance and they are minimal by $15 \mathrm{kpc}$.

The right panel in Figure 3 shows the line emissivity as a function of source proximity. The emissivity at $5 \mathrm{kpc}$ is at least 2 orders of magnitude higher than the purely collisional case at all temperatures. The boost is about 2 orders of magnitude at $20 \mathrm{kpc}$, and remains within a factor of a few even at $100 \mathrm{kpc}$.

Note that less bright sources lead to more modest effects (Section 4.1).

\section{INTENSITY GRIDS}

Because the vast majority of the hyperfine lines are optically thin for a wide range of conditions, it is beneficial to compile intensity grids as a function of the gas density and temperature. Such calculations are presented in the following. 

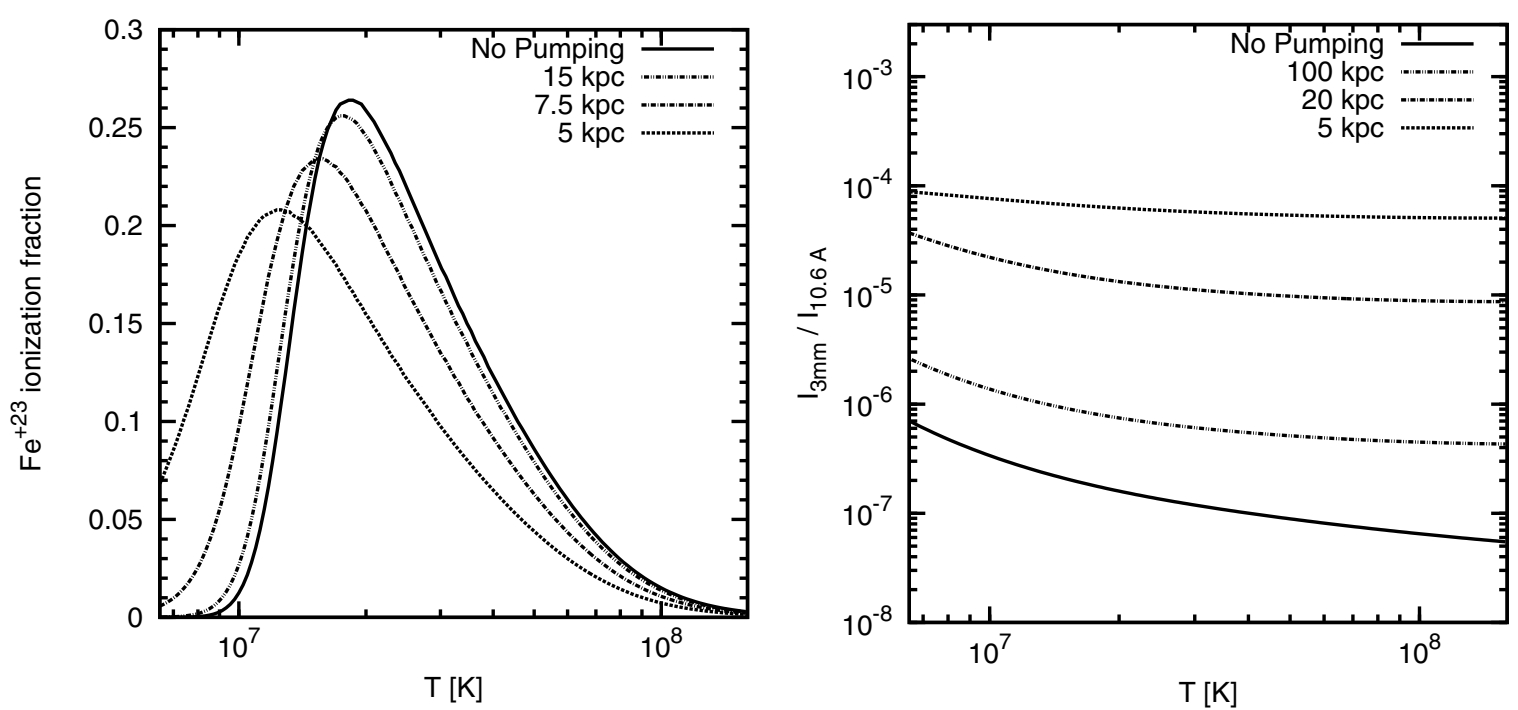

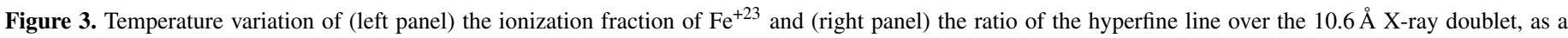

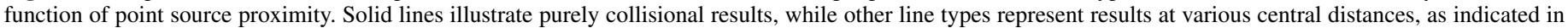
the keys.

At each grid point, continuum-corrected line intensities are computed for a unit volume of gas of definite density and temperature, exposed to the CMB. The temperature varies in the range $10^{4}-10^{8} \mathrm{~K}$, in which the relevant ions are expected to be most abundant, while the density varies in the range $10^{-3}-10^{+3} \mathrm{~cm}^{-3}$.

Figure 4 presents the intensity contour maps. The quantity plotted in the contour maps below is the intensity integrated over all angles and the line profile, and has units of log flux. The maps are sorted in order of descending peak intensity.

All maps follow a similar outline. At a given temperature, the intensity increases with the density. The critical density of the two-level system may be defined as

$$
n_{\mathrm{e}, \mathrm{c}}=\frac{g_{l} A_{\mathrm{ul}} T^{1 / 2}}{8.629 \times 10^{-6} \Omega^{\mathrm{eff}}}\left(1+\eta_{\nu}\right)
$$

where $g_{l}$ is the statistical weight of the lower hyperfine level, and $\eta_{v}$ is the CMB photon occupation number at the line frequency. Obviously, the exact value of the critical density for a given line depends on the transition probability, as well as the effective collision strength. Sodium-like lines tend to have critical densities below our lowest limit and vary as the first power of the density. On the other hand, hydrogenic lines tend to have critical densities above our highest limit and vary as the square of the density. Finally, the critical densities for most lithium-like lines fall within the covered range and vary as $n^{2}$ at low densities and as $n$ at high densities.

On the other hand, at a given density, the intensity rises quickly with temperature, reaches a peak at some characteristic value at which the ionization fraction is maximum, and then declines. For hydrogenic atoms, the line intensity contours at higher temperatures are convex (positive second derivative), while for lithium-like and sodium-like atoms the contours are concave and, in some cases, bend sharply to higher densities. In addition, the Li-like and Na-like contours of second- and third-row elements possess a secondary intensity peak at higher temperatures.

These types of behavior at high temperatures may be understood as follows. First, the slow variation of the contours of hydrogenic atoms arises from the balance between collisional ionization and radiative recombination between the hydrogenic ions and the corresponding bare nuclei. In this situation, the electron density drops out of the balance equation, and the density ratio of these ionization stages is equal to the ratio of the recombination coefficient over the collisional ionization coefficient, which varies very slowly with temperature.

On the other hand, in lithium-like and sodium-like ions, such a balance cannot be achieved because the ion can be further ionized. The ion abundance decreases quickly with temperature, at a rate regulated by the ionization potential. As a result, the intensity contours for lithium-like ions of fourth-row elements decrease gently, while for second- and third-row elements, as well as for all sodium-like ions, the contours decrease more sharply.

As the temperature increases, the closed shell is progressively ionized. Some of the released electrons may recombine with lower stage ions, e.g., electrons released from He-like carbon may recombine with Li-like carbon. The second intensity peak seen in lithium-like and sodium-like ions of second- and third-row elements arises due to the fact that dielectronic recombination with these electrons is more important than radiative recombination. However, the deeper potential wells of fourth-row elements disfavor the excitations necessary for dielectronic recombination, and moderate its importance.

\subsection{Normalizing to the $20 \AA \mathrm{X}$-Ray Bump}

As an observational aid, we express the intensity of the coronal lines relative to the intensity of the line complex at $20 \AA$ (18-28 A, or $0.45-0.70 \mathrm{keV})$, including the bremsstrahlung continuum.

For reference, the intensity contours of the X-ray complex are shown in Figure 5, along with the spectra of various physical conditions of interest. At high temperatures, the contours are nearly vertical due to the fact that the intensity for both the continuum and the line emission varies with the square of the density, with a slow dependence on temperature.

Figure 6 shows the contours of the normalized line flux, for those ions whose ionization fraction obtains its highest value at temperatures above $10^{5.4} \mathrm{~K}$. Below the critical density, the contours are have only a temperature dependence. More 

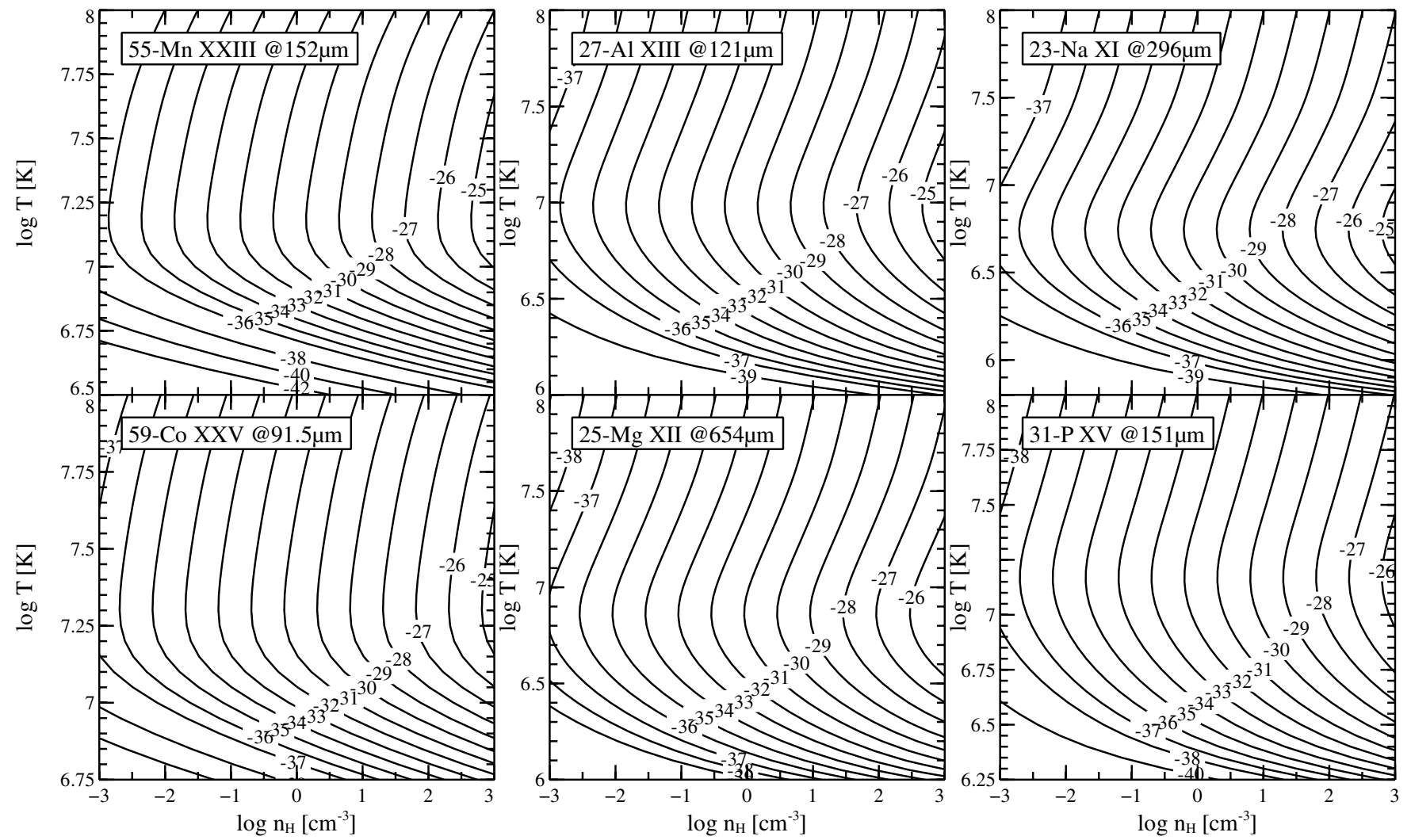

Figure 4. Log intensity maps for the coronal hyperfine lines in order of descending peak intensity from left to right, and from top to bottom.

(The complete figure set (14 images) is available in the online journal.)
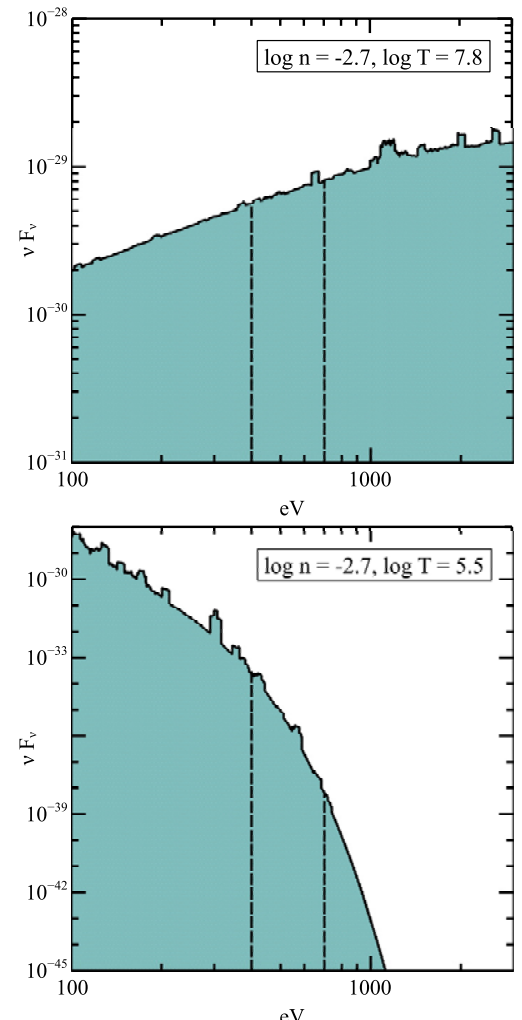
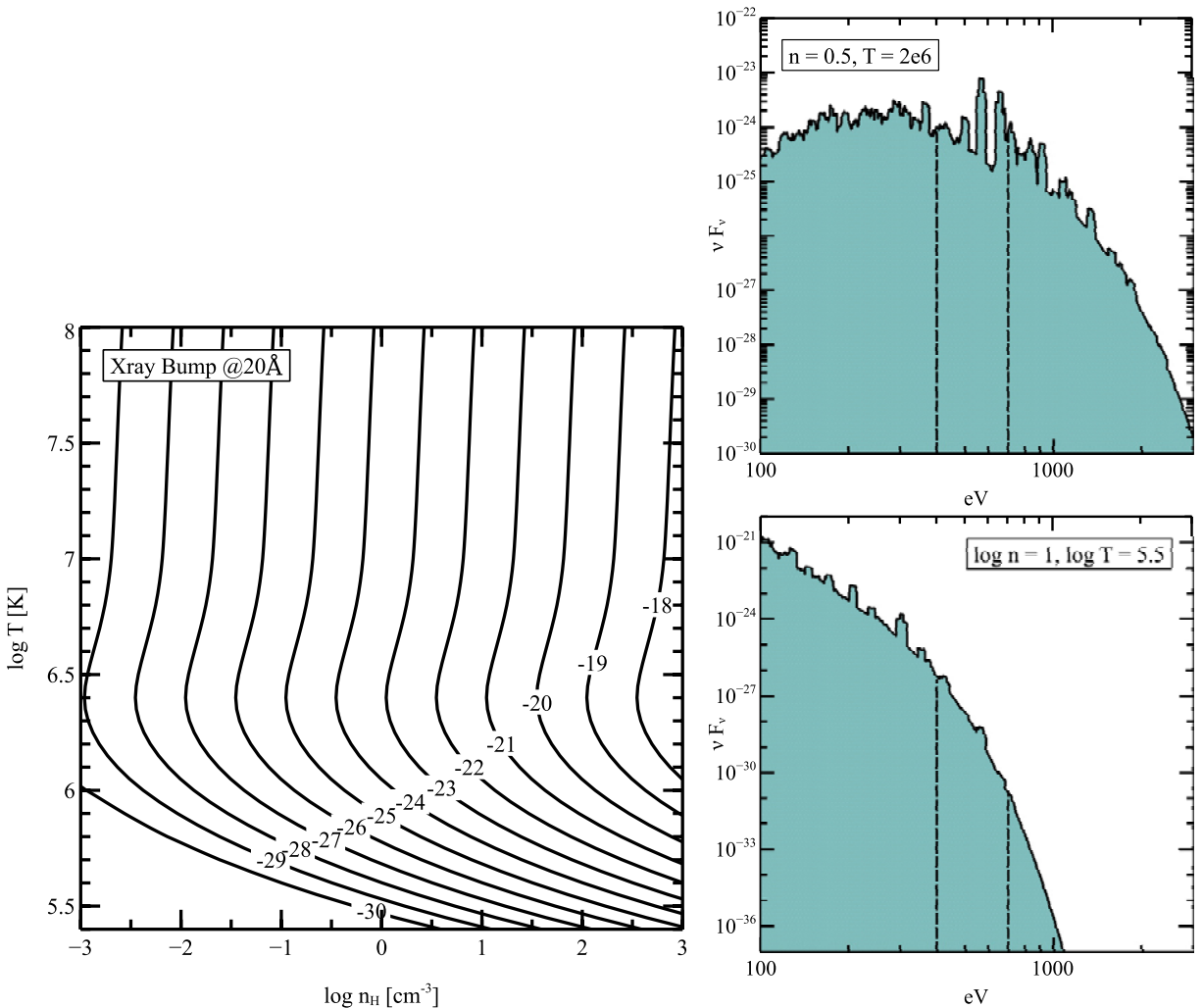

Figure 5. Center: Intensity contours of the X-ray complex at $20 \AA$ (see the text), including the bremsstrahlung continuum. Left and right: X-ray spectra in the range $0.1-3 \mathrm{keV}$ for various points in the grid, as indicated in the legends. The density and temperature for the top right spectrum are similar to the conditions of the hot gas in the Extended Orion Nebula (Güdel et al. 2008). The spectra are at higher resolution than typically attainable in X-ray observations.

(A color version of this figure is available in the online journal.) 

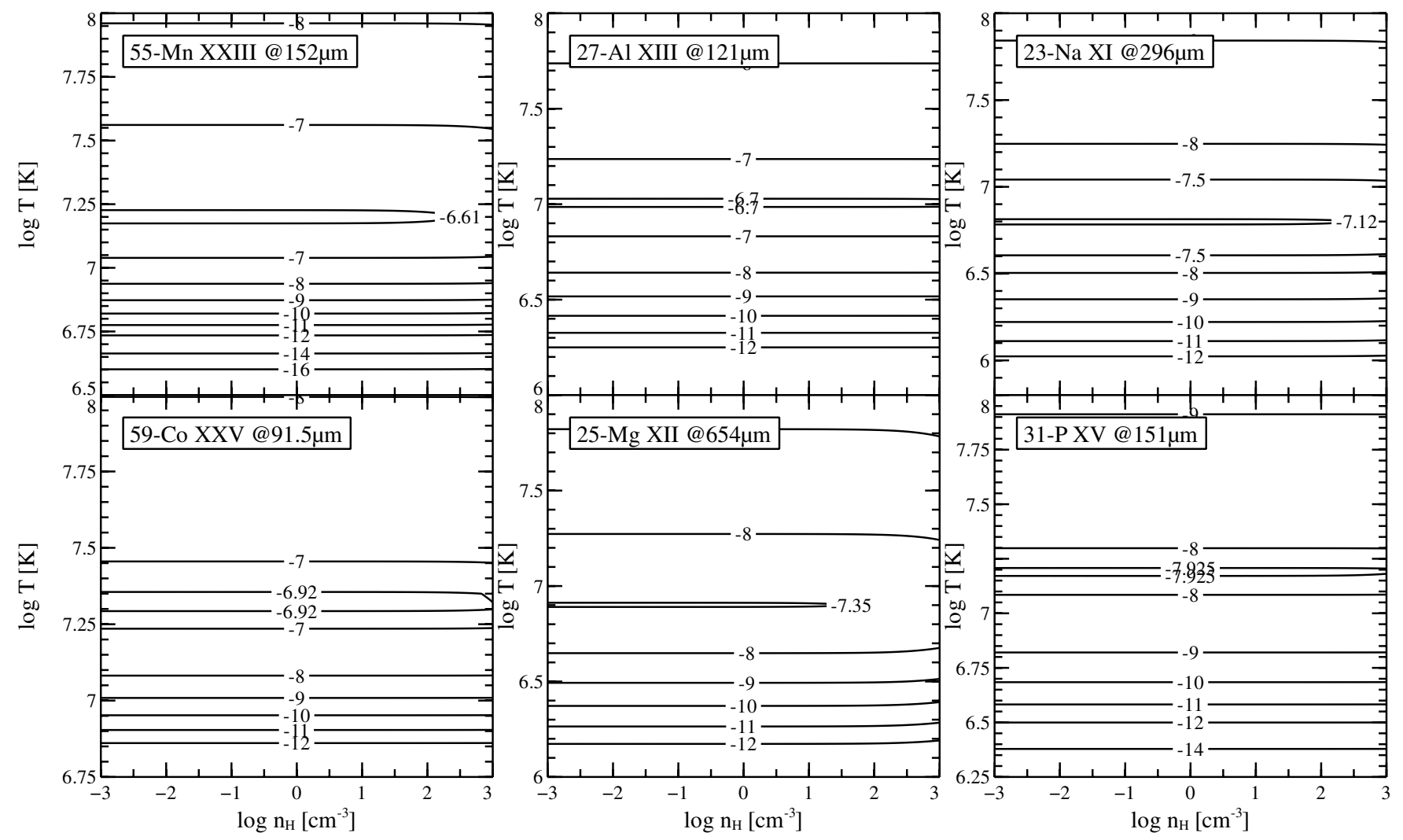

Figure 6. Intensity maps for the coronal hyperfine lines normalized by the intensity of the X-ray line complex. The images are presented in the same order as Figure 4 . (The complete figure set (11 images) is available in the online journal.)

complicated contour outlines are manifest above the critical density. Accordingly, most hydrogenic lines and lithium-like lines of heavy elements have density-independent contours, contrary to lines that arise from shallower ionization potentials.

\section{HYPERFINE LINE PREDICTIONS}

In this section, we use Cloudy to compute the hyperfine line intensities that arise from hot gas in the Perseus and Virgo galaxy clusters, as well as in the Orion Nebula.

Cloudy reports the emissivity integrated over the line profile and over all angles. We convert this to the spectral emissivity at the line center by using the formula

$$
j_{v}=\frac{j}{4 \pi^{3 / 2} \Delta v_{D}},
$$

where $\Delta v_{D}$ is the Doppler line width. The $4 \pi$ term accounts for the angular dependence, while the remainder is the profile at the line center, that is, $\phi\left(v_{0}\right)=\left\{\sqrt{\pi} \Delta v_{D}\right\}^{-1}$.

We deduce the profile at the line center as follows. We assume that the line follows the Voigt profile, balanced by the competition between natural (radiative and pressure) broadening on one hand, and Doppler broadening on the other. This is encapsulated by the parameter $\alpha=\left(\gamma^{\mathrm{rad}}+\gamma^{\mathrm{col}}\right) /\left(4 \pi \Delta v_{D}\right)$, where the $\gamma$ values are the rates of radiative $\left(\gamma^{\mathrm{rad}}=A_{\mathrm{ul}}\right)$ and collisional damping. Because pressure broadening at the line core is best described by the impact approximation, we take the damping rate equal to the collisional deexcitation rate, $\gamma^{\mathrm{col}}=C_{\mathrm{ul}}$. The parameter, then, obtains vanishingly small values $(\alpha \ll 1)$, and the (unnormalized) Voigt function at the line center is roughly 1 , leading to the equation above.
Note that in the following, the Doppler width does not include a turbulent component, so our intensity estimates in the context of galaxy clusters should be treated as upper limits.

\subsection{Galaxy Clusters}

Realistic line predictions require accurate descriptions of the distribution of the ICM density, temperature, and metallicity with distance from the cluster center. For that purpose, we employ Chandra, XMM-Newton, and ROSAT observations as described in detail below.

For all hyperfine lines, we compute the (CMB-corrected) emissivity radial profiles. All lines are optically thin when integrated through the cluster diameters, with the highest optical depth in Perseus having a value of about $10^{-4}$. The transfer of the hyperfine lines can then be ignored, and intensity profiles can be computed by simply integrating the emissivity profiles along the line of sight.

In the tables below, for each line we present three estimates for the intensity: at the peak of the intensity profile, for a 0.1 square arc-minute fiducial aperture centered on the cluster center, and for the entire cluster volume. Brightness temperatures are provided for lines with $\lambda>1 \mathrm{~mm}$ as well. The line flux is also expressed in units of the $\mathrm{X}$-ray continuum flux.

\subsubsection{Perseus Galaxy Cluster}

We model the ICM in Perseus with the aid of the XMMNewton observations of Churazov et al. (2004) for the plasma within the central $200 \mathrm{kpc}$, and of the ROSAT observations of Ettori et al. (1998) for the plasma in the cluster outskirts. For the metallicity in the outskirts, we use the average metallicity of the outermost bins of the XMM-Newton observation (0.48 solar). 

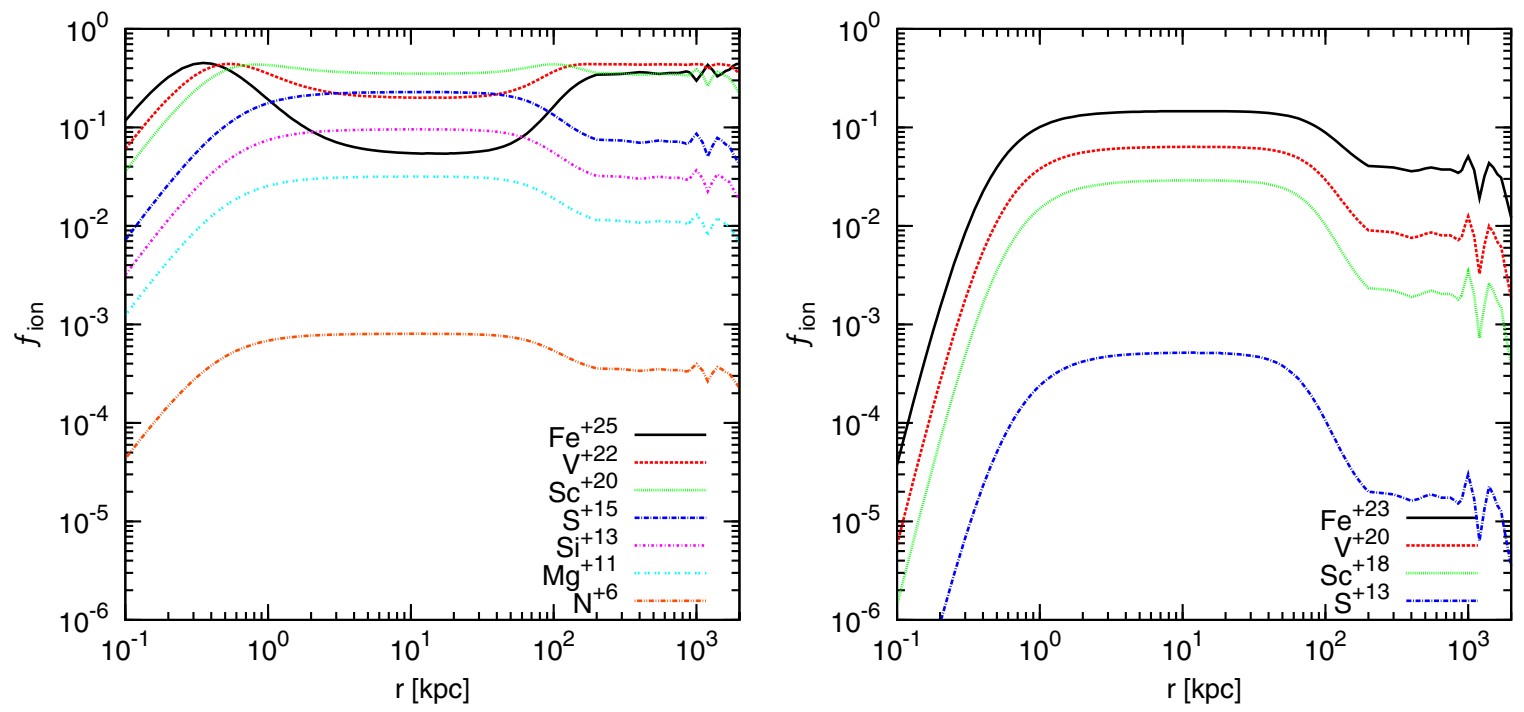

Figure 7. Example ionization fraction profiles for hydrogenic (left panel) and lithium-like (right panel) ions. The effects of the AGN radiation are prominent within the central $2 \mathrm{kpc}$. Note that the temperature profile rises beyond $100 \mathrm{kpc}$ from a central floor of about $3 \mathrm{keV}$ to about $6.5 \mathrm{keV}$ at $900 \mathrm{kpc}$. The structure seen around $1 \mathrm{Mpc}$ is due to uncertainties in the input ROSAT temperature profile.

(A color version of this figure is available in the online journal.)
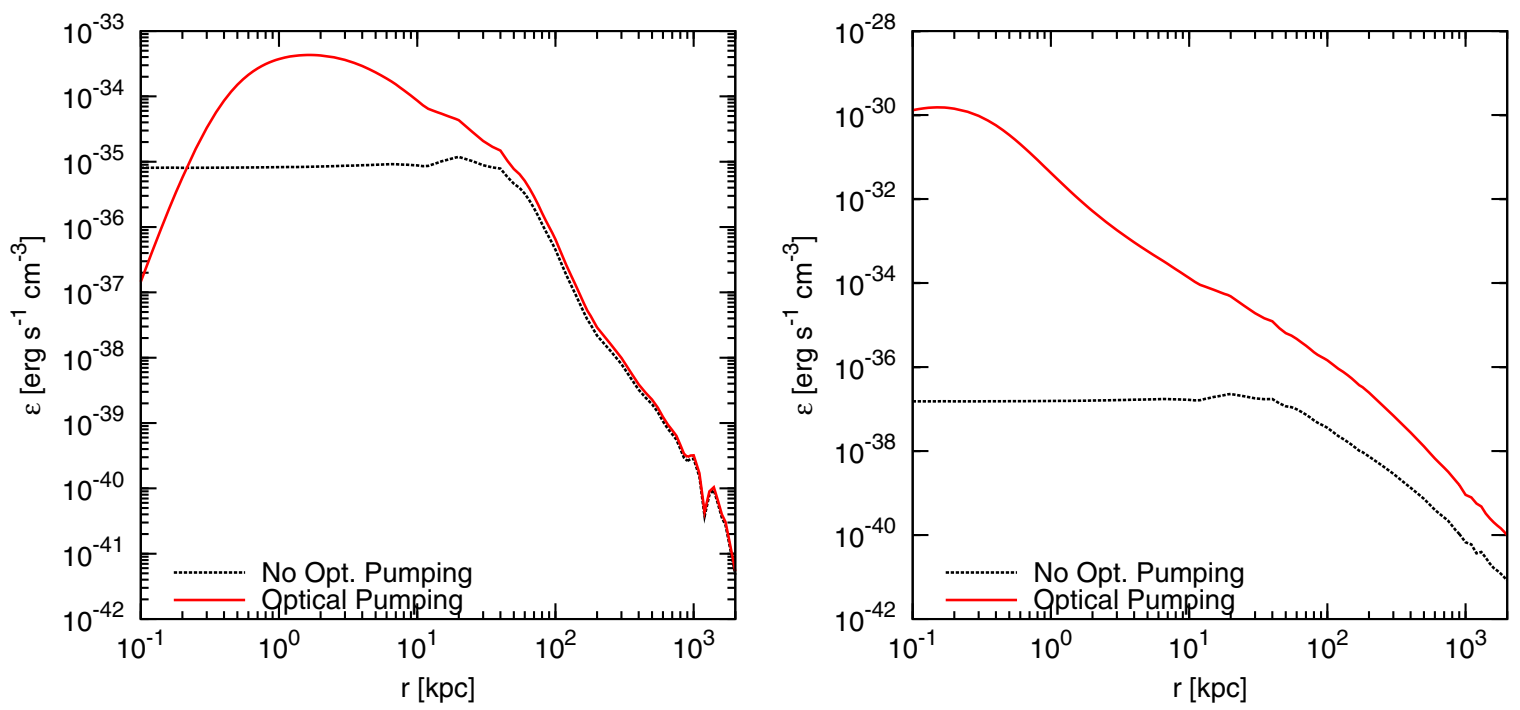

Figure 8. Comparison of the predicted emissivity profile of the ${ }^{57} \mathrm{Fe}$ XXIV $\lambda 3.068 \mathrm{~mm}$ (left panel), and the ${ }^{57} \mathrm{Fe}$ XXVI $\lambda 348 \mu \mathrm{m}$ (right panel) lines in Perseus, with the effects of optical pumping accounted for and ignored.

(A color version of this figure is available in the online journal.)

Note that the substructure at the cluster center revealed with Chandra (Fabian et al. 2006) is not expected to significantly alter our conclusions, and has not been accounted for in these calculations.

As suggested in Section 2.2, the presence of the AGNs can greatly affect the ionization structure of the ICM near the cluster center. Figure 7 illustrates the effects of the intense radiation on the ionization structure of the plasma. The ions shown were selected because they are relevant to X-ray spectroscopy obtained from cool core clusters (Peterson \& Fabian 2006). In the absence of the ionizing background, the ion fractions are constant within the temperature core of the cluster $(r \lesssim$ $50 \mathrm{kpc}$ ). By contrast, the AGN radiation field significantly alters the ionization structure of the gas within the central $2 \mathrm{kpc}$, reducing the fractional densities of species with shallow ionization potentials and enhancing those with deep potentials. In our calculations, the densities of hydrogenic ions of elements with $21 \leqslant Z \leqslant 27$ are increased by up to 1 order of magnitude.

Because the effect is localized to the vicinity of the AGNs, the altered ionization fractions do not greatly affect our estimates for (projected) line intensities. However, the AGN may significantly impact the ionization structure of the interstellar medium near the center of the brightest cluster galaxy NGC 1275. A detailed exploration of this possibility is beyond the scope of this paper.

Line emissivities are affected by the AGNs through the ionization fractions, as well as through optical pumping. Figure 8 illustrates that for the physical conditions prevalent at the cluster core, the emissivity of the ${ }^{57} \mathrm{Fe}$ XxIV line reaches a boost of $\sim 50$ 
Table 5

Perseus Brightest Lines

\begin{tabular}{|c|c|c|c|c|c|c|c|c|}
\hline Species & $\begin{array}{c}\lambda \\
(\mathrm{cm}) \\
(2)\end{array}$ & $\begin{array}{c}I_{v}^{\max } \\
\left(\text { mJy ster }^{-1} \text { ) }\right. \\
(3)\end{array}$ & $\begin{array}{c}T_{b}^{\max } \\
(\mathrm{mK}) \\
(4)\end{array}$ & $\begin{array}{c}I_{v}^{\mathrm{apr}} \\
\left(\mathrm{mJy} \mathrm{ster}^{-1}\right) \\
(5)\end{array}$ & $\begin{array}{c}T_{b}^{\mathrm{apr}} \\
(\mathrm{mK}) \\
(6)\end{array}$ & $\begin{array}{c}I_{v}^{\mathrm{tot}} \\
\left(\mathrm{mJy} \mathrm{ster}^{-1}\right) \\
(7)\end{array}$ & $\begin{array}{c}T_{b}^{\mathrm{tot}} \\
(\mathrm{mK}) \\
(8)\end{array}$ & $\begin{array}{c}F / F_{\text {cont }} \\
\text { (9) }\end{array}$ \\
\hline${ }^{27} \mathrm{Al}$ XIII & $1.210 \mathrm{E}-02$ & $2.646 \mathrm{E}+07$ & $\ldots$ & $5.223 \mathrm{E}+06$ & $\ldots$ & $4.471 \mathrm{E}+02$ & $\ldots$ & $8.268 \mathrm{E}-07$ \\
\hline${ }^{31} \mathrm{P} \times V$ & $1.510 \mathrm{E}-02$ & $1.259 \mathrm{E}+07$ & $\ldots$ & $2.701 \mathrm{E}+06$ & $\ldots$ & $2.305 \mathrm{E}+02$ & $\ldots$ & $3.197 \mathrm{E}-07$ \\
\hline${ }^{55} \mathrm{Mn}$ XXIII & $1.520 \mathrm{E}-02$ & $6.317 \mathrm{E}+06$ & $\ldots$ & $2.017 \mathrm{E}+06$ & $\ldots$ & $3.226 \mathrm{E}+02$ & $\ldots$ & $1.783 \mathrm{E}-07$ \\
\hline${ }^{57} \mathrm{Fe} \mathrm{XXVI}$ & $3.480 \mathrm{E}-02$ & $3.372 \mathrm{E}+07$ & $\ldots$ & $1.219 \mathrm{E}+06$ & $\ldots$ & $1.261 \mathrm{E}+02$ & $\ldots$ & $4.619 \mathrm{E}-08$ \\
\hline${ }^{57} \mathrm{Fe}$ XXIV & $3.068 \mathrm{E}-01$ & $1.896 \mathrm{E}+06$ & $6.4532 \mathrm{E}-03$ & $1.197 \mathrm{E}+06$ & $4.0747 \mathrm{E}-03$ & $4.533 \mathrm{E}+02$ & $1.5431 \mathrm{E}-06$ & $5.167 \mathrm{E}-09$ \\
\hline${ }^{59} \mathrm{CoxxV}$ & $9.150 \mathrm{E}-03$ & $2.388 \mathrm{E}+06$ & $\ldots$ & $6.887 \mathrm{E}+05$ & $\ldots$ & $1.142 \mathrm{E}+02$ & $\ldots$ & $9.763 \mathrm{E}-08$ \\
\hline${ }^{25} \mathrm{Mg}$ XII & $6.540 \mathrm{E}-02$ & $2.689 \mathrm{E}+06$ & $\ldots$ & $6.160 \mathrm{E}+05$ & $\ldots$ & $6.212 \mathrm{E}+01$ & $\ldots$ & $1.875 \mathrm{E}-08$ \\
\hline${ }^{35} \mathrm{Cl}$ XVII & $2.130 \mathrm{E}-02$ & $2.459 \mathrm{E}+06$ & $\ldots$ & $5.079 \mathrm{E}+05$ & $\ldots$ & $4.624 \mathrm{E}+01$ & $\ldots$ & $4.011 \mathrm{E}-08$ \\
\hline${ }^{39} \mathrm{~K}$ XIX & $3.170 \mathrm{E}-02$ & $2.551 \mathrm{E}+06$ & $\ldots$ & $4.601 \mathrm{E}+05$ & $\ldots$ & $4.514 \mathrm{E}+01$ & $\ldots$ & $2.313 \mathrm{E}-08$ \\
\hline${ }^{29} \mathrm{Si}$ XIV & $3.790 \mathrm{E}-02$ & $1.987 \mathrm{E}+06$ & $\ldots$ & $4.172 \mathrm{E}+05$ & $\ldots$ & $4.029 \mathrm{E}+01$ & $\ldots$ & $2.035 \mathrm{E}-08$ \\
\hline${ }^{53} \mathrm{Cr}$ XXIV & $1.270 \mathrm{E}-02$ & $6.458 \mathrm{E}+06$ & $\ldots$ & $3.461 \mathrm{E}+05$ & $\ldots$ & $3.628 \mathrm{E}+01$ & $\ldots$ & $3.726 \mathrm{E}-08$ \\
\hline${ }^{23} \mathrm{Na} \mathrm{XI}$ & $2.960 \mathrm{E}-02$ & $1.250 \mathrm{E}+06$ & $\ldots$ & $2.396 \mathrm{E}+05$ & $\ldots$ & $2.527 \mathrm{E}+01$ & $\ldots$ & $1.680 \mathrm{E}-08$ \\
\hline${ }^{33} \mathrm{~S}$ XVI & $3.270 \mathrm{E}-02$ & $9.451 \mathrm{E}+05$ & $\ldots$ & $2.044 \mathrm{E}+05$ & $\ldots$ & $1.939 \mathrm{E}+01$ & $\ldots$ & $1.083 \mathrm{E}-08$ \\
\hline${ }^{14} \mathrm{~N}$ VII & $5.652 \mathrm{E}-01$ & $2.535 \mathrm{E}+05$ & $2.9289 \mathrm{E}-03$ & $1.675 \mathrm{E}+05$ & $1.9355 \mathrm{E}-03$ & $6.317 \mathrm{E}+01$ & $7.2983 \mathrm{E}-07$ & $7.919 \mathrm{E}-10$ \\
\hline${ }^{37} \mathrm{Cl} \mathrm{XVII}$ & $2.550 \mathrm{E}-02$ & $6.552 \mathrm{E}+05$ & $\ldots$ & $1.368 \mathrm{E}+05$ & $\ldots$ & $1.267 \mathrm{E}+01$ & $\ldots$ & $8.778 \mathrm{E}-09$ \\
\hline${ }^{49} \mathrm{Ti}$ XXII & $8.350 \mathrm{E}-03$ & $1.157 \mathrm{E}+06$ & $\ldots$ & $1.065 \mathrm{E}+05$ & $\ldots$ & $1.089 \mathrm{E}+01$ & $\ldots$ & $1.814 \mathrm{E}-08$ \\
\hline
\end{tabular}

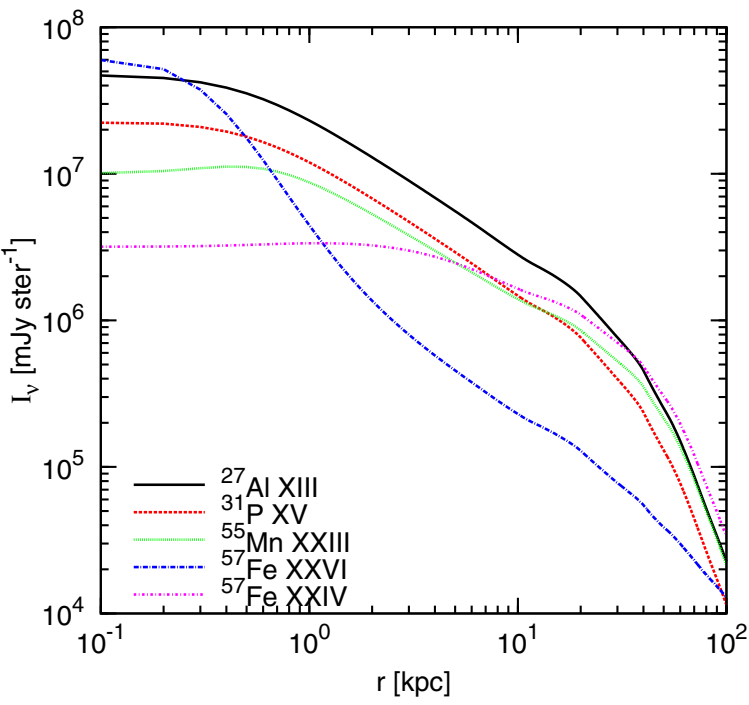

Figure 9. Intensity profiles within (projected) $100 \mathrm{kpc}$ of NGC 1275 for the five brightest hyperfine lines.

(A color version of this figure is available in the online journal.)

within the central $2 \mathrm{kpc}$, but plummets close to the center due to the modified ion densities. On the other hand, the ${ }^{57} \mathrm{Fe}$ XXVI line is boosted by about 7 orders of magnitude near the AGNs, by about 3 orders of magnitude at $10 \mathrm{kpc}$, and by about 1 order of magnitude even in the cluster outskirts. Clearly, optical pumping by the central AGN can affect the emissivities of certain lines throughout the cluster volume.

Table 5 lists the brightest lines in Perseus in order of decreasing average intensity within the aperture. The lines are in the millimeter or submillimeter part of the spectrum, and they are all enhanced by optical pumping, similar to the iron lines.

Figure 9 presents the intensity profiles for the five brightest lines in Perseus, that is, their emissivities integrated along the line of sight as a function of the projected distance from the cluster center. The diversity in profile shapes is caused by the intense radiation field of the AGNs.

\subsubsection{Virgo Galaxy Cluster}

We utilize the density and temperature fits of Churazov et al. (2008) to model the ICM in the Virgo cluster. We also make use of the detailed fits of Million et al. (2011) to describe the elemental abundance profiles of several elements ( $\mathrm{Ne}, \mathrm{Mg}, \mathrm{Si}$, $\mathrm{S}, \mathrm{Ar}, \mathrm{Ca}, \mathrm{Fe}$, and $\mathrm{Ni}$ ), and employ an average of these profiles for other metals.

Because the radiation field of 3C 274 is more dilute than NGC 1275 (Figure 13), the ionization structure of the plasma, and line emissivities are much more modestly affected. The emissivity boost at $1 \mathrm{kpc}$ amounts to about 2 for the ${ }^{57} \mathrm{Fe}$ XXIV line, and about 1 order of magnitude for the ${ }^{27} \mathrm{Al}$ XIII line. These are the two brightest lines in Virgo, according to Table 6.

However, the radiation field may still affect the ion fractions of hydrogenic species with $Z \geqslant 17$, as shown in the left panel of Figure 10. In addition, the emissivity of the ${ }^{57} \mathrm{Fe}$ XXVI line is boosted by several orders of magnitude in the vicinity of the AGNs (right panel in Figure 10), and by a factor of two at the cluster outskirts.

The intensity profiles of the five brightest lines are shown in Figure 11. While their central values are comparable, the intensities at $5 \%$ of the virial radius $(12 \mathrm{kpc})$ vary by almost 2 orders of magnitude. This behavior is the opposite of that in Perseus.

We have also examined the emissivity that arises from the arms of cool gas emanating from the AGNs (Forman et al. 2007). We have used the emission measure maps of Werner et al. (2010), along with their estimates for the line-of-sight depth of the arms for various values of the inclination angle from the sightline. Our calculations suggest that due to the limited depth along the sightline, the arms increase line intensities by up to $1 \%$.

\subsection{Extended Orion Nebula}

Güdel et al. (2008) discovered two bubbles of hot gas in the Extended Orion Nebula, southwest of the Huygens region. These bubbles are thought to be the wind of the Trapezium stars, which is being channeled into the Orion-Eridanus Superbubble, 
Table 6

Virgo Brightest Lines

\begin{tabular}{|c|c|c|c|c|c|c|c|c|}
\hline Species & $\begin{array}{c}\lambda \\
(\mathrm{cm}) \\
(2)\end{array}$ & $\begin{array}{c}I_{v}^{\max } \\
\left(\mathrm{mJy} \mathrm{ster}^{-1}\right) \\
(3)\end{array}$ & $\begin{array}{c}T_{b}^{\max } \\
(\mathrm{mK}) \\
(4)\end{array}$ & $\begin{array}{c}I_{v}^{\mathrm{apr}} \\
\left(\mathrm{mJy} \mathrm{ster}^{-1}\right) \\
(5)\end{array}$ & $\begin{array}{c}T_{b}^{\mathrm{apr}} \\
(\mathrm{mK}) \\
(6)\end{array}$ & $\begin{array}{c}I_{v}^{\mathrm{tot}} \\
\left(\mathrm{mJy} \mathrm{ster}^{-1}\right) \\
(7)\end{array}$ & $\begin{array}{c}T_{b}^{\mathrm{tot}} \\
(\mathrm{mK}) \\
(8)\end{array}$ & $\begin{array}{c}F / F_{\text {cont }} \\
(9)\end{array}$ \\
\hline${ }^{57} \mathrm{Fe}$ XXIV & $3.068 \mathrm{E}-01$ & $8.388 \mathrm{E}+06$ & $2.8553 \mathrm{E}-02$ & $4.343 \mathrm{E}+06$ & $1.4782 \mathrm{E}-02$ & $1.220 \mathrm{E}+03$ & $4.1524 \mathrm{E}-06$ & $1.352 \mathrm{E}-08$ \\
\hline${ }^{27} \mathrm{Al}$ XIII & $1.210 \mathrm{E}-02$ & $2.378 \mathrm{E}+07$ & $\ldots$ & $3.636 \mathrm{E}+06$ & $\ldots$ & $2.452 \mathrm{E}+02$ & $\ldots$ & $4.162 \mathrm{E}-07$ \\
\hline${ }^{25} \mathrm{Mg}$ XII & $6.540 \mathrm{E}-02$ & $1.377 \mathrm{E}+07$ & $\ldots$ & $2.131 \mathrm{E}+06$ & $\ldots$ & $1.488 \mathrm{E}+02$ & $\ldots$ & $4.689 \mathrm{E}-08$ \\
\hline${ }^{55} \mathrm{Mn}$ XXIII & $1.520 \mathrm{E}-02$ & $8.652 \mathrm{E}+06$ & $\ldots$ & $1.992 \mathrm{E}+06$ & $\ldots$ & $6.828 \mathrm{E}+02$ & $\ldots$ & $1.275 \mathrm{E}-07$ \\
\hline${ }^{31} \mathrm{P} \times \mathrm{V}$ & $1.510 \mathrm{E}-02$ & $1.113 \mathrm{E}+07$ & $\ldots$ & $1.623 \mathrm{E}+06$ & $\ldots$ & $1.006 \mathrm{E}+02$ & $\ldots$ & $1.389 \mathrm{E}-07$ \\
\hline${ }^{29} \mathrm{Si} \mathrm{XIV}$ & $3.790 \mathrm{E}-02$ & $1.078 \mathrm{E}+07$ & $\ldots$ & $1.616 \mathrm{E}+06$ & $\ldots$ & $9.750 \mathrm{E}+01$ & $\ldots$ & $5.697 \mathrm{E}-08$ \\
\hline${ }^{14} \mathrm{~N}$ VII & $5.652 \mathrm{E}-01$ & $1.271 \mathrm{E}+06$ & $1.4688 \mathrm{E}-02$ & $8.449 \mathrm{E}+05$ & $9.7607 \mathrm{E}-03$ & $1.333 \mathrm{E}+02$ & $1.5403 \mathrm{E}-06$ & $2.880 \mathrm{E}-09$ \\
\hline${ }^{23} \mathrm{Na} \mathrm{XI}$ & $2.960 \mathrm{E}-02$ & $4.855 \mathrm{E}+06$ & $\ldots$ & $7.471 \mathrm{E}+05$ & $\ldots$ & $5.494 \mathrm{E}+01$ & $\ldots$ & $3.787 \mathrm{E}-08$ \\
\hline${ }^{59} \mathrm{Co} \mathrm{Xxv}$ & $9.150 \mathrm{E}-03$ & $2.800 \mathrm{E}+06$ & $\ldots$ & $5.349 \mathrm{E}+05$ & $\ldots$ & $1.901 \mathrm{E}+02$ & $\ldots$ & $5.491 \mathrm{E}-08$ \\
\hline${ }^{33} \mathrm{~S}$ XVI & $3.270 \mathrm{E}-02$ & $2.682 \mathrm{E}+06$ & $\ldots$ & $3.775 \mathrm{E}+05$ & $\ldots$ & $2.648 \mathrm{E}+01$ & $\ldots$ & $1.446 \mathrm{E}-08$ \\
\hline${ }^{35} \mathrm{Cl}$ XVII & $2.130 \mathrm{E}-02$ & $2.606 \mathrm{E}+06$ & $\ldots$ & $3.452 \mathrm{E}+05$ & $\ldots$ & $2.833 \mathrm{E}+01$ & $\ldots$ & $1.971 \mathrm{E}-08$ \\
\hline${ }^{53} \mathrm{Cr}$ XXII & $1.130 \mathrm{E}-01$ & $9.341 \mathrm{E}+05$ & $4.3137 \mathrm{E}-04$ & $2.131 \mathrm{E}+05$ & $9.8419 \mathrm{E}-05$ & $5.368 \mathrm{E}+01$ & $2.4789 \mathrm{E}-08$ & $1.868 \mathrm{E}-09$ \\
\hline${ }^{39} \mathrm{~K}$ XVII & $2.940 \mathrm{E}-01$ & $3.290 \mathrm{E}+05$ & $1.0284 \mathrm{E}-03$ & $1.760 \mathrm{E}+05$ & $5.5011 \mathrm{E}-04$ & $4.377 \mathrm{E}+01$ & $1.3683 \mathrm{E}-07$ & $6.916 \mathrm{E}-10$ \\
\hline${ }^{39} \mathrm{~K}$ XIX & $3.170 \mathrm{E}-02$ & $1.487 \mathrm{E}+06$ & $\ldots$ & $1.658 \mathrm{E}+05$ & $\ldots$ & $1.777 \mathrm{E}+01$ & $\ldots$ & $6.026 \mathrm{E}-09$ \\
\hline${ }^{37} \mathrm{Cl}$ XVII & $2.550 \mathrm{E}-02$ & $8.809 \mathrm{E}+05$ & $\ldots$ & $1.166 \mathrm{E}+05$ & $\ldots$ & $9.491 \mathrm{E}+00$ & $\ldots$ & $5.412 \mathrm{E}-09$ \\
\hline
\end{tabular}

a sparse structure of hot plasma, elongated about $100 \mathrm{kpc}$ along the sightline, and seen to the south of the Orion Nebula. Of particular relevance to our discussion is the discovery of radioactivity due to the rare ${ }^{26} \mathrm{Al}$ in the Superbubble (Diehl 2002), which supports the scenario of replenishment over short timescales, and suggests the presence of other isotopes at significant fractions.

We have modeled the north and South cavities adopting their best-fit physical conditions and an average path length of $2 \mathrm{pc}$. The density and temperature for the north cavity are $0.47 \mathrm{~cm}^{-3}$, and $1.73 \mathrm{MK}$, respectively. For the South cavity, the physical conditions are $0.23 \mathrm{~cm}^{-3}$, and $2.08 \mathrm{MK}$.

The results of these calculations are presented in Table 7. Column 5 lists the line surface brightness in units of the surface brightness of the continuum (shown in the top right panel of Figure 5). Both lines arise from species whose ionization fractions peak around $2 \mathrm{MK}$.

Note that rough estimates for these lines may also be obtained by using the contour plots of Figure 6. First, we calculate the continuum surface brightness. From Figure 3 of Güdel et al. (2008), we approximate the $0.4-0.7 \mathrm{keV}$ count rate as 0.12 and 0.3 counts $\mathrm{s}^{-1}$ for the north and south cavities, respectively. We assume that the average energy of each photon in this band is $0.5 \mathrm{keV}$, and take the relevant effective area of the PN detector on board XMM-Newton to be $1000 \mathrm{~cm}^{2}$. We adopt the parallactic distance to Orion ( $410 \mathrm{pc}$; Reid et al. 2009) to convert the projected cavity areas to solid angles. The continuum surface brightness turns out to be $\sim 7 \mathrm{E}-8$ and $\sim 3 \mathrm{E}-8 \mathrm{erg} \mathrm{s}^{-1} \mathrm{~cm}^{-2} \mathrm{ster}^{-1}$, for the north and south cavities.

The two brightest among the lines of Figure 6 for the plasma conditions in the two cavities are indeed ${ }^{27} \mathrm{Al} \mathrm{XI} \lambda 1.206 \mathrm{~mm}$, and ${ }^{14} \mathrm{~N}$ VII $\lambda 5.625 \mathrm{~mm}$, with respective surface brightness of $1 \mathrm{E}-6$ and $1 \mathrm{E}-7$ relative to the continuum. These rough estimates are in a factor of 3 agreement with the results listed in Column 5 of Table 7 . The frequency broadening for these lines is $\sim 50 \mathrm{MHz}$, and $\sim 14 \mathrm{MHz}$, leading to intensities within factors of a few from the values listed in Column 3 of Table 7. These discrepancies are in part due to the very approximate continuum surface brightness values adopted here. However, our approach illustrates that the surface brightness ratios of Figure 6 provide a convenient alternative to modeling when rough estimates of optically thin line intensities are desired.
Table 7

EON Brightest Lines

\begin{tabular}{|c|c|c|c|c|}
\hline Species & $\begin{array}{c}\lambda \\
(\mathrm{cm}) \\
(2)\end{array}$ & $\begin{array}{c}I_{v} \\
\left(\mathrm{mJy} \mathrm{ster}^{-1}\right) \\
(3)\end{array}$ & $\begin{array}{c}T_{b} \\
(\mathrm{mK}) \\
(4)\end{array}$ & $\begin{array}{c}\mathrm{I} / I_{\text {cont }} \\
\quad(5)\end{array}$ \\
\hline \multicolumn{5}{|c|}{ EON North } \\
\hline${ }^{27} \mathrm{Al} \mathrm{XI}$ & $1.206 \mathrm{E}-01$ & $3.327 \mathrm{E}+05$ & $1.7525 \mathrm{E}-04$ & $1.0333 \mathrm{E}-06$ \\
\hline${ }^{14} \mathrm{~N}$ VII & $5.652 \mathrm{E}-01$ & $3.164 \mathrm{E}+05$ & $3.6604 \mathrm{E}-03$ & $2.9118 \mathrm{E}-07$ \\
\hline \multicolumn{5}{|c|}{ EON South } \\
\hline${ }^{27} \mathrm{Al}$ XI & $1.206 \mathrm{E}-01$ & $1.032 \mathrm{E}+05$ & $5.4344 \mathrm{E}-05$ & $5.5171 \mathrm{E}-07$ \\
\hline${ }^{14} \mathrm{~N}$ VII & $5.652 \mathrm{E}-01$ & $9.846 \mathrm{E}+04$ & $1.1390 \mathrm{E}-03$ & $1.5601 \mathrm{E}-07$ \\
\hline
\end{tabular}

We have also modeled the emission that arises from the Superbubble itself, adopting for the density, temperature, and sightline depth the values $0.015 \mathrm{~cm}^{-3}, 3.25 \mathrm{MK}$, and $150 \mathrm{kpc}$, respectively (Guo et al. 1995). However, the signal remains below $1 \mu \mathrm{K}$ and is not discussed further here. In fact, the signal would remain below that limit even if the isotope ratios were enhanced as expected for Wolf-Rayet stellar winds (Meynet et al. 2001).

\section{DISCUSSION AND CONCLUSIONS}

\subsection{Galaxy Clusters}

Syunyaev \& Churazov (1984) and D’Cruz \& Sarazin (C. L. Sarazin 2013, private communication) predicted the brightness temperature of the ${ }^{57} \mathrm{Fe}$ XXIV line to be around $1 \mathrm{mK}$. Our calculations draw a more pessimistic picture. This is in part due to the more accurate description of the X-ray emitting gas in cool cluster cores, obtained with recent X-ray observations. In particular, in previous work on Perseus, the density has likely been overestimated by a factor of a few, which accounts for at least 1 order of magnitude of the discrepancy, in the absence of optical pumping. Even when the effects of the AGN radiation are incorporated, the maximum intensity remains well below previous predictions.

Our calculations suggest that the ${ }^{57} \mathrm{Fe}$ line will be brighter in Virgo than in Perseus, with a brightness temperature in our fiducial aperture of only $15 \mu \mathrm{K}$. A $3 \sigma$ detection would require 

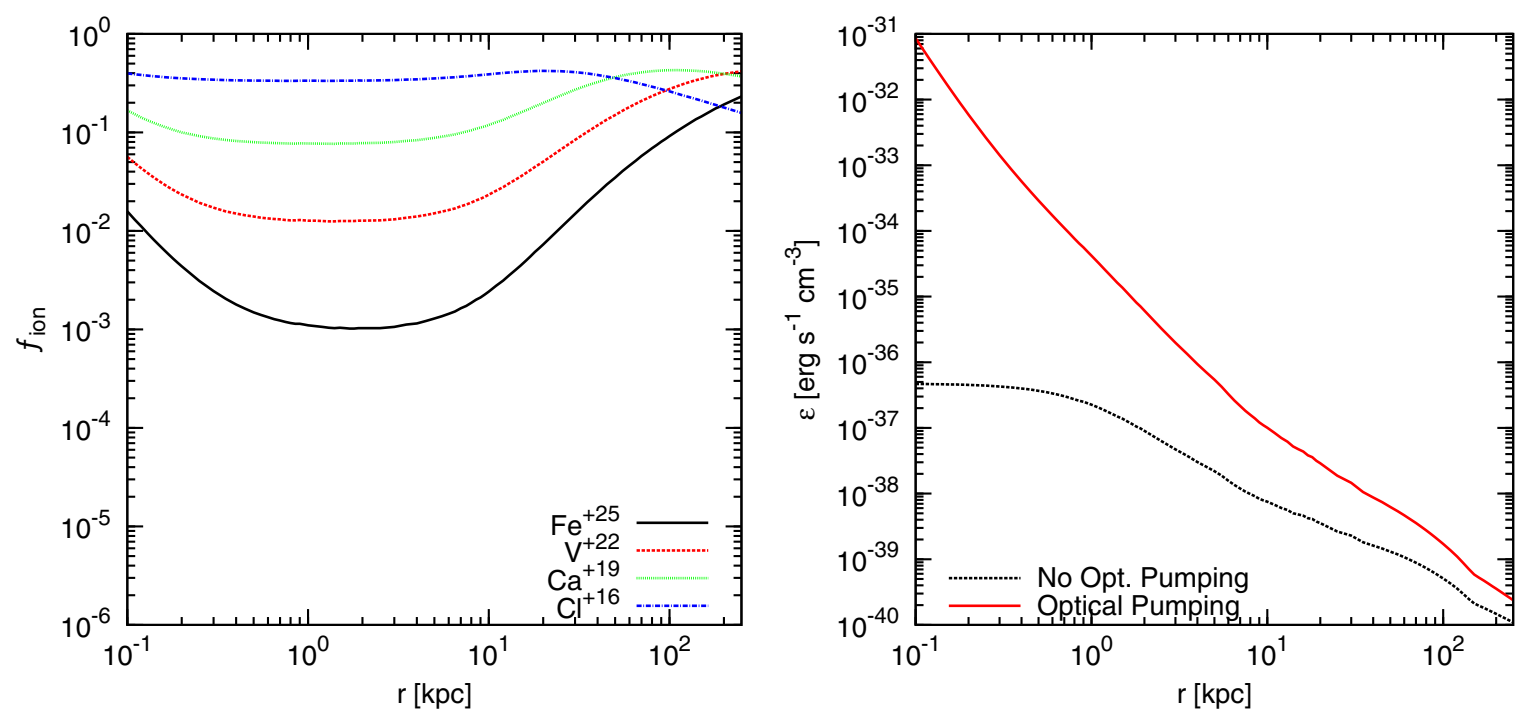

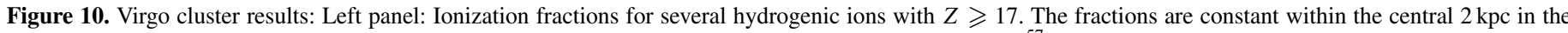

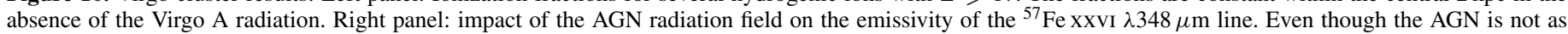
bright as Perseus A, the emissivity boost is at least one order of magnitude within the central $10 \mathrm{kpc}$.

(A color version of this figure is available in the online journal.)

about $\sim 600 \mathrm{hr}$ exposure with the full Atacama Large Millimeter Array $\left(A L M A ; T_{b} \sim 20 \mu \mathrm{K}\right.$ within an $8^{\prime \prime}$ beam).

Our calculations suggest that the ${ }^{57} \mathrm{Fe}$ XXIV line is not always the brightest hyperfine line, as suggested by previous work. Both clusters produce a number of lines (Al XIII $\lambda 121 \mu \mathrm{m}$, $\mathrm{PXV} \lambda 151 \mu \mathrm{m}$, and Mn XXIII $\lambda 152 \mu \mathrm{m})$ with intensities within a factor of three of the ${ }^{57} \mathrm{Fe}$ XXIV line. In fact, in Perseus, these submillimeter lines are brighter. Clearly, our understanding of nucleosynthesis in galaxy clusters would benefit from submillimeter spectrometers.

Clusters at higher redshift are not necessarily more likely to be observed. Because the majority of emission arises from the cluster core, the brightness temperature of the entire cluster drops dramatically by about 4-5 orders of magnitude when the integration extends over the entire cluster volume. An optimal solid angle will depend on the line intensity profile, as well as on the cluster redshift.

Interestingly, we find that the AGNs can strongly affect the plasma ionization structure within the central $1 \mathrm{kpc}$. The density of high ionization stages is enhanced, leading to a reduction of line emission due to lower stages. This occurs only for high- $Z$ elements about M87. It is possible that this effect could have important implications for the interpretation of spectra of bright AGNs in the X-ray, as well as at longer wavelengths.

\subsection{Orion Nebula}

The X-ray cavities in the Extended Orion Nebula are thought to have formed by hot plasma leaking out of the Huygens region of the Orion Nebula. Hyperfine line detections in these regions would help constrain isotopic ratios in the molecular cloud, and could shed some light into nucleosynthesis in OB stars. However, both cavities in the EON are quite faint, with the north cavity being about three times brighter than the south. The brightest line in terms of intensity is Al XI $\lambda 1.206 \mathrm{~mm}$, although in terms of brightness temperature the N VII line is substantially more promising. The brightness temperature of this line is about $3 \mu \mathrm{K}$, comparable to Perseus. This line is inaccessible to groundbased telescopes due to atmospheric opacity.

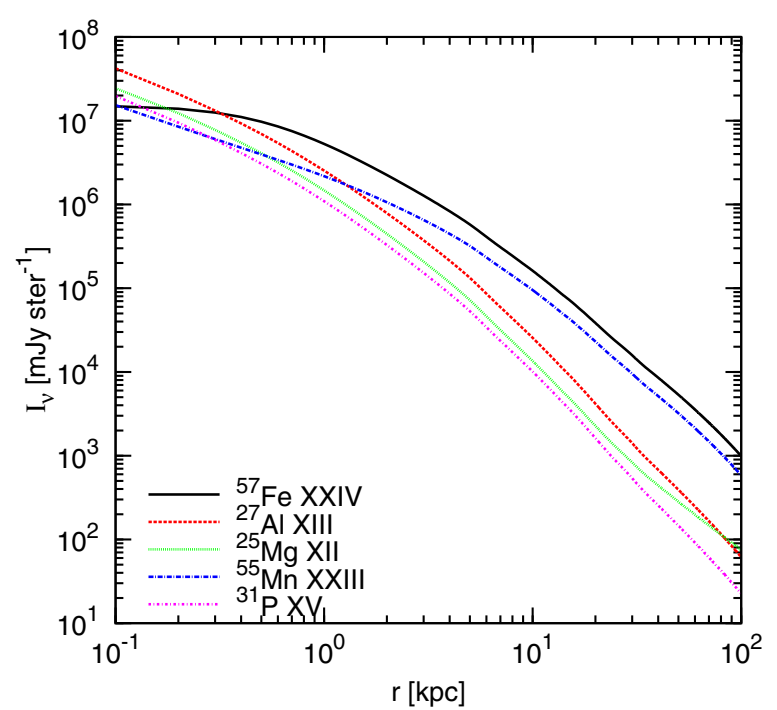

Figure 11. Intensity profiles for the five brightest lines in the Virgo cluster. (A color version of this figure is available in the online journal.)

\section{SUMMARY}

We have investigated the possibility of observing the hyperfine lines of Syunyaev \& Churazov (1984) that arise from high ionization species in hot plasmas.

Our calculations are carried out with the spectral synthesis code CLOUDY, and incorporate direct, resonant, and indirect excitations due to collisional and radiative processes. In addition, radiative and dielectronic recombinations are included self-consistently. The framework provided by CLOUDY allows for the uniform treatment of all hyperfine lines.

Our calculations incorporate stimulated radiative transitions by the CMB, with corrections performed according to Chatzikos et al. (2013). We find that bright point sources, such as Perseus A, can significantly alter the ionization structure of a plasma at close proximity, as well line emissivity at larger distances. Less bright point sources, such as Virgo A, can affect the ionization 
fraction of highly ionized heavy elements, and the emissivities of lines with wavelengths in the range $200-1100 \mu \mathrm{m}$. It may be the case that the AGNs radiation affects optical lines, as well. If this turns out to be true, it will likely affect the interpretation of the nuclear spectra of Perseus A, as well as of other systems.

We have computed hyperfine line intensities over grids covering a wide range of density and temperature, as an aid to observations. We have also expressed these line intensities in units of the X-ray continuum in the range $18-28 \AA$, which includes the line complex due to carbon, nitrogen and oxygen ions.

We have improved upon the estimates of Syunyaev \& Churazov (1984) and D'Cruz \& Sarazin (2013, private communication) on the brightness of the ${ }^{57} \mathrm{Fe}$ XXIV line. Our results are not encouraging: in Perseus, the line is more than two orders of magnitude fainter than previously thought. The line is brighter in Virgo by a factor of a few, but, having a brightness temperature of $\sim 20 \mu \mathrm{K}$, it would still require long exposure times with $A L M A$ even for a marginal detection. Our theoretical calculations are consistent with the observationally motivated estimates of Liang et al. (1997).

In addition, submillimeter lines with intensities within a factor of 3-4 of the iron line are produced by both clusters, which may provide alternatives to hyperfine line detections.

Similar conclusions hold for the Extended Orion Nebula. The high density of the cavity plasma is balanced by the low sightline depth, leading to brightness temperatures comparable to those in Perseus. However, the brightest line is the N vII $\lambda 5.625 \mathrm{~mm}$ line which is inaccessible from ground-based observatories.

Obviously, our understanding of nucleosynthesis and turbulent velocity fields in galaxy clusters and molecular clouds would benefit from submillimeter instrumentation to resolve these hyperfine emission lines, as well as from millimeter space observatories to detect the aforementioned N VII line. Our results, then, echo in part the conclusions of Sunyaev \& Docenko (2007), who find ${ }^{14} \mathrm{~N}$ vII $\lambda 5.625 \mathrm{~mm}$ to be the best hyperfine tracer of hot plasmas in the Milky Way.

M.C. thanks Craig Sarazin for useful conversations. G.J.F. acknowledges support by NSF (1108928 and 1109061), NASA (10-ATP10-0053, 10-ADAP10-0073, and NNX12AH73G), and STScI (HST-AR-12125.01, HST-AR-13245, GO-12560, and HST-GO-12309).

\section{APPENDIX A \\ IMPROVED FITS TO DIRECT AND RESONANT EXCITATION DATA}

In this section, we present our improvements upon the fits of Goddard \& Ferland (2003, GF03) to the ZS00 data. Overall, with the present approach the ZSO0 total collision strengths may be approximated to $10 \%$ or better for temperatures $10^{6} \mathrm{~K}$ or higher and $25 \%$ for temperatures in the range $10^{5}-10^{6} \mathrm{~K}$. For comparison, GF03 report approximations of $30 \%$ or better.

While GF03 fit the total effective collision strengths as a function of temperature, we model the contribution of each excitation mechanism (direct and resonant through $n=2$ and $n=3$ ) to the total collision strength of each species and isosequence independently.
All temperature distributions may be fit with a log-normal function of the form

$$
Y^{\mathrm{eff}}(T)=N\left(\frac{T}{10^{6} T_{\mathrm{s} 1}}\right)^{C_{1}} \exp \left\{-\left|\frac{\log _{10}\left(T /\left(10^{6} T_{\mathrm{s} 1}\right)\right)}{T_{\mathrm{s} 2}}\right|^{C_{2}}\right\},
$$

adopting a fiducial error of $3 \%$ in the reported collision strength values. Note that the $\mathrm{ZS} 00$ data are evaluated at 11 temperature points (10 for iron), sufficient to constrain the best-fit values of a 5 parameter functional form. The accuracy of these fits is $\leqslant 3 \%$ for the direct excitations, and resonances through $n=2$ in Li-like isosequences, $\leqslant 20 \%$ for resonances through $n=3$ in Li-like ions, and $\leqslant 30 \%$ for resonances through $n=2$ in hydrogenic ions. The values and errors of the best-fit parameters to each of these distributions are presented in Table 8.

The constants that appear in this expression $\left(N, T_{\mathrm{s} 1}, C_{1}\right.$, $T_{\mathrm{s} 2}$, and $C_{2}$ ) vary with the species, isosequence, and excitation mechanism under consideration. Across an isosequence and excitation process, these constants display a strong dependence on the nuclear charge, and occasionally a minor dependence on the nuclear spin. This dependence on the nuclear properties has been modeled with good accuracy across each isosequence and excitation mechanism with the use of the auxiliary functions

$$
\begin{gathered}
f(x)=A\left\{\log _{10}(x / B)\right\}^{C}, \\
g(x)=A \exp \left\{-(x / B)^{C}\right\}, \\
h(x)=A \exp \left\{(x / B)^{C}\right\}, \\
k(x)=A(x / B)^{C}\{\exp (x / B)\}^{D}, \\
l(x)=A(x / B)^{C} \exp \left\{(x / B)^{D}\right\}, \\
m(x)=A(x / B)^{C} \exp \left\{-(x / B)^{D}\right\},
\end{gathered}
$$

as shown in Table 9. Note that these forms are not independent of each other, but are employed here for convenience. While they are constrained by the five Li-like species, the two hydrogenic species are not sufficient to constrain the fits in any reliable fashion.

In the Li-like ions, parameters with undefined uncertainties have been held fixed to improve the goodness-of-fit measure, as it was found that their exact values did not significantly affect the agreement of the best-fit function with the available data points.

By contrast, to circumvent the lack of sufficient data in the hydrogenic sequences, it was assumed that the scaling ought to be similar to that of the Li-like isosequence. In detail, we have assumed that the scaling with nuclear properties (parameter $B$ ) is the same across isosequences, allowing only the normalization and power-law exponent (parameters $A$ and $C$ ) to vary. When present, the power law associated with the exponential (parameter $D$ ) was held fixed at the Li-like value.

Note also that owing to the similarity in the shape and amplitude of their profiles, we have adopted the functional forms of $n=3$ resonances of Li-like ions for the $n=2$ resonances of the hydrogenic ions.

A comparison between the present work and the fits of GF03 is presented in Figure 12. In all but two cases, the fitting function 
Table 8

Best-Fit Parameters to ZS00 Data

\begin{tabular}{|c|c|c|c|c|c|c|c|c|c|c|c|c|}
\hline $\begin{array}{l}\text { Ion } \\
\text { (1) }\end{array}$ & $\begin{array}{c}N_{\text {dof }} \\
\text { (2) }\end{array}$ & $\begin{array}{l}\chi_{\text {dof }}^{2} \\
\text { (3) }\end{array}$ & $\begin{array}{c}N \\
(4)\end{array}$ & $\begin{array}{l}\sigma_{N} \\
(5)\end{array}$ & $\begin{array}{l}T_{\mathrm{s} 1} \\
(6)\end{array}$ & $\begin{array}{c}\sigma_{T_{\mathrm{s} 1}} \\
(7)\end{array}$ & $\begin{array}{l}C_{1} \\
(8)\end{array}$ & $\begin{array}{c}\sigma_{C_{1}} \\
(9)\end{array}$ & $\begin{array}{c}T_{\mathrm{S} 2} \\
(10)\end{array}$ & $\begin{array}{l}\sigma_{T_{\mathrm{s} 2}} \\
(11)\end{array}$ & $\begin{array}{c}C_{2} \\
(12)\end{array}$ & $\begin{array}{l}\sigma_{C_{2}} \\
(13)\end{array}$ \\
\hline \multicolumn{13}{|c|}{ Direct (Background) Excitations } \\
\hline${ }^{13} \mathrm{C}^{+5}$ & 7 & 0.00313728 & 0.0463139 & 0.0002839 & 0.207503 & 0.02996 & -0.0475049 & 0.00721 & 1.86432 & 0.04121 & 2.56332 & 0.04545 \\
\hline${ }^{14} \mathrm{~N}^{+6}$ & 7 & 0.00155694 & 0.0610659 & 0.0002067 & 0.223362 & 0.02193 & -0.039488 & 0.003979 & 1.93797 & 0.03046 & 2.67975 & 0.03559 \\
\hline${ }^{23} \mathrm{Na}^{+8}$ & 7 & 0.0126283 & 0.0735341 & 0.002088 & 0.178673 & 0.06534 & -0.0816228 & 0.03259 & 1.68484 & 0.06735 & 2.27324 & 0.06218 \\
\hline${ }^{25} \mathrm{Mg}^{+9}$ & 7 & 0.00893359 & 0.0928638 & 0.001721 & 0.185271 & 0.05118 & -0.0715463 & 0.02126 & 1.72992 & 0.05934 & 2.34265 & 0.05738 \\
\hline${ }^{27} \mathrm{Al}^{+10}$ & 7 & 0.00585586 & 0.0768643 & 0.0009545 & 0.191162 & 0.04052 & -0.0633176 & 0.01421 & 1.77343 & 0.05083 & 2.40811 & 0.05123 \\
\hline${ }^{29} \mathrm{Si}^{+11}$ & 7 & 0.00314582 & 0.0166136 & 0.0001278 & 0.198736 & 0.02943 & -0.056951 & 0.008833 & 1.81119 & 0.03837 & 2.4691 & 0.04003 \\
\hline${ }^{57} \mathrm{Fe}^{+23}$ & 6 & 0.0139415 & 0.00374383 & 0.0001595 & 1.78522 & 0.7198 & -0.109575 & 0.04304 & 1.61725 & 0.0603 & 2.21178 & 0.05548 \\
\hline \multicolumn{13}{|c|}{$n=2$ Resonant Excitations } \\
\hline${ }^{13} \mathrm{C}^{+5}$ & 7 & 60.4757 & 0.00286807 & 0.0003172 & 3.42741 & 0.4091 & 0.336739 & 0.1964 & 0.627927 & 0.03002 & 3.65514 & 0.1326 \\
\hline${ }^{14} \mathrm{~N}^{+6}$ & 7 & 106.616 & 0.00481214 & 0.0007836 & 3.36366 & 0.3964 & 0.801919 & 0.2618 & 0.577669 & 0.02999 & 3.65531 & 0.1519 \\
\hline${ }^{23} \mathrm{Na}^{+8}$ & 7 & 0.0233641 & 0.126021 & 0.01115 & 3.79216 & 0.3447 & -0.96184 & 0.004376 & 1.64596 & 0.03367 & 3.56222 & 0.06541 \\
\hline${ }^{25} \mathrm{Mg}^{+9}$ & 7 & 0.00987751 & 0.157279 & 0.01532 & 4.34866 & 0.4322 & -0.972123 & 0.003537 & 1.81967 & 0.03689 & 3.32988 & 0.06 \\
\hline${ }^{27} \mathrm{Al}^{+10}$ & 7 & 0.0329427 & 0.133248 & 0.01365 & 3.95003 & 0.4163 & -0.959629 & 0.005621 & 1.61544 & 0.03793 & 3.45352 & 0.07018 \\
\hline${ }^{29} \mathrm{Si}^{+11}$ & 7 & 0.0201666 & 0.0344953 & 0.002928 & 3.95752 & 0.3462 & -0.958917 & 0.004656 & 1.65588 & 0.03101 & 3.34559 & 0.05451 \\
\hline${ }^{57} \mathrm{Fe}^{+23}$ & 6 & 0.00708709 & 0.0146527 & 0.0007875 & 1.88574 & 0.1189 & -0.836432 & 0.008784 & 2.67792 & 0.08321 & 1.44764 & 0.02205 \\
\hline \multicolumn{13}{|c|}{$n=3$ Resonant Excitations } \\
\hline${ }^{23} \mathrm{Na}^{+8}$ & 7 & 4.9649 & 0.0216931 & 0.00228 & 3.74735 & 0.5855 & -0.561302 & 0.07659 & 0.86893 & 0.03629 & 3.19419 & 0.09567 \\
\hline${ }^{25} \mathrm{Mg}^{+9}$ & 7 & 8.8006 & 0.0330297 & 0.003101 & 3.47036 & 0.5493 & -0.442042 & 0.09474 & 0.831089 & 0.03501 & 3.24333 & 0.1095 \\
\hline${ }^{27} \mathrm{Al}^{+10}$ & 7 & 13.1543 & 0.0313274 & 0.002508 & 3.23321 & 0.4854 & -0.31384 & 0.1071 & 0.800671 & 0.03228 & 3.31426 & 0.1206 \\
\hline${ }^{29} \mathrm{Si}^{+11}$ & 7 & 17.0828 & 0.00739256 & 0.0005303 & 3.0921 & 0.4331 & -0.193367 & 0.1142 & 0.778544 & 0.03019 & 3.39744 & 0.1274 \\
\hline${ }^{57} \mathrm{Fe}^{+23}$ & 6 & 0.925002 & 0.00115868 & 0.0001332 & 31.1566 & 4.592 & -0.731262 & 0.04629 & 0.965965 & 0.03518 & 2.95329 & 0.07596 \\
\hline
\end{tabular}

Table 9

Correlations of Best-Fit Parameters to ZS00 Data

\begin{tabular}{|c|c|c|c|c|c|c|c|c|c|c|c|}
\hline $\begin{array}{l}\text { Parameter } \\
\text { (1) }\end{array}$ & $\begin{array}{c}\text { Function } \\
\text { (2) }\end{array}$ & $\begin{array}{c}N_{\text {dof }} \\
(3)\end{array}$ & $\begin{array}{l}\chi_{\mathrm{dof}}^{2} \\
(4)\end{array}$ & $\begin{array}{c}\mathrm{A} \\
(5)\end{array}$ & $\begin{array}{l}\sigma_{A} \\
(6)\end{array}$ & $\begin{array}{l}\text { B } \\
\text { (7) }\end{array}$ & $\begin{array}{l}\sigma_{B} \\
(8)\end{array}$ & $\begin{array}{l}C \\
(9)\end{array}$ & $\begin{array}{c}\sigma_{C} \\
(10)\end{array}$ & $\begin{array}{c}\mathrm{D} \\
(11)\end{array}$ & $\begin{array}{c}\sigma_{D} \\
(12)\end{array}$ \\
\hline \multicolumn{12}{|c|}{ Li-like Direct (Background) Excitations } \\
\hline $\mathrm{N}$ & $f(\sqrt{I} / Z)$ & 2 & $7.308 \mathrm{e}+00$ & $1.286 \mathrm{e}-02$ & $1.850 \mathrm{e}-02$ & $4.481 \mathrm{e}-03$ & $2.954 \mathrm{e}-03$ & 5.03933 & 1.215 & $\ldots$ & $\ldots$ \\
\hline$T_{s 1}$ & $h(Z)$ & 2 & $4.266 \mathrm{e}-04$ & $1.708 \mathrm{e}-01$ & $1.821 \mathrm{e}-03$ & $2.143 e+01$ & $1.383 \mathrm{e}-01$ & 4.41718 & 0.1231 & $\ldots$ & $\ldots$ \\
\hline$C_{1}$ & $l(Z)$ & 1 & $3.519 \mathrm{e}-05$ & $-2.339 \mathrm{e}-02$ & $5.039 \mathrm{e}-04$ & $2.404 \mathrm{e}+01$ & $3.609 \mathrm{e}-01$ & -1.59178 & 0.05401 & 6.54585 & 0.8688 \\
\hline$T_{s 2}$ & $m(Z)$ & 2 & $2.650 \mathrm{e}-04$ & $2.364 \mathrm{e}+00$ & $7.109 \mathrm{e}-02$ & $3.125 \mathrm{e}+01$ & $9.468 \mathrm{e}-01$ & 0.323085 & 0.01819 & 6.1953 & 1.305 \\
\hline$C_{2}$ & $m(Z)$ & 1 & $5.809 \mathrm{e}-06$ & $3.217 \mathrm{e}+00$ & $9.603 e-03$ & $2.969 \mathrm{e}+01$ & $1.390 \mathrm{e}-01$ & 0.349369 & 0.001352 & 8.3886 & 0.3214 \\
\hline \multicolumn{12}{|c|}{ Li-like $n=2$ Resonances } \\
\hline $\mathrm{N}$ & $f(I / Z)$ & 2 & $8.700 \mathrm{e}-01$ & $5.249 \mathrm{e}-02$ & $3.626 \mathrm{e}-02$ & $5.186 \mathrm{e}-03$ & $3.286 \mathrm{e}-03$ & 2.26828 & 0.6926 & $\ldots$ & $\ldots$ \\
\hline$T_{s 1}$ & $g(Z)$ & 2 & $6.460 \mathrm{e}-01$ & $4.743 \mathrm{e}+00$ & $3.093 e-01$ & $2.678 \mathrm{e}+01$ & $\ldots$ & 2.33347 & 0.6295 & $\ldots$ & $\ldots$ \\
\hline$C_{1}$ & $g(Z)$ & 2 & $2.756 e+00$ & $-9.720 \mathrm{e}-01$ & $1.524 \mathrm{e}-02$ & $4.127 e+01$ & $1.192 \mathrm{e}+01$ & 4.10155 & 2.742 & $\ldots$ & $\ldots$ \\
\hline$T_{s 2}$ & $\frac{Z}{\sqrt{I}} h(\sqrt{I} / Z)$ & 2 & $4.368 \mathrm{e}+00$ & $6.338 \mathrm{e}-02$ & $7.959 \mathrm{e}-03$ & $1.100 \mathrm{e}-01$ & $1.049 \mathrm{e}-02$ & 1.57284 & 0.3833 & $\ldots$ & $\ldots$ \\
\hline$C_{2}$ & $g(Z)$ & 2 & $2.926 \mathrm{e}+00$ & $3.569 \mathrm{e}+00$ & $2.206 \mathrm{e}-01$ & $2.664 \mathrm{e}+01$ & $2.603 e-01$ & 4.19725 & 1.812 & $\ldots$ & $\ldots$ \\
\hline \multicolumn{12}{|c|}{ Li-like $n=3$ Resonances } \\
\hline $\mathrm{N}$ & $f(I / Z)$ & 2 & $1.319 \mathrm{e}+00$ & $2.717 \mathrm{e}-02$ & $1.615 e-03$ & $1.597 \mathrm{e}-02$ & $1.042 \mathrm{e}-03$ & 1.25218 & 0.1487 & $\ldots$ & $\ldots$ \\
\hline$T_{s 1}$ & $k(Z)$ & 2 & $3.740 \mathrm{e}-02$ & $7.168 \mathrm{e}-03$ & $1.646 \mathrm{e}-03$ & $1.000 \mathrm{e}+01$ & $\ldots$ & -8.85041 & 0.4891 & 6.47427 & 0.2666 \\
\hline$C_{1}$ & $l(Z)$ & 2 & $2.240 \mathrm{e}+01$ & $-3.762 \mathrm{e}-01$ & $7.083 e-02$ & $1.000 \mathrm{e}+01$ & $\ldots$ & -7.94404 & 0.9693 & 2.20888 & 0.09759 \\
\hline$T_{s 2}$ & $k(Z)$ & 2 & $4.878 \mathrm{e}-03$ & $2.933 \mathrm{e}-01$ & $6.833 e-03$ & $1.000 \mathrm{e}+01$ & $\ldots$ & -1.88971 & 0.04903 & 1.15294 & 0.02704 \\
\hline$C_{2}$ & $k(Z)$ & 2 & $5.896 \mathrm{e}-02$ & $5.920 \mathrm{e}+00$ & $4.141 \mathrm{e}-01$ & $1.000 \mathrm{e}+01$ & $\ldots$ & 1.05838 & 0.1436 & -0.656385 & 0.07999 \\
\hline \multicolumn{12}{|c|}{ H-like Direct (Background) Excitations } \\
\hline $\mathrm{N}$ & $f(\sqrt{I} / Z)$ & 0 & $\ldots$ & $8.499 \mathrm{e}-03$ & $\ldots$ & $4.481 \mathrm{e}-03$ & $\ldots$ & 4.83542 & $\ldots$ & $\ldots$ & $\ldots$ \\
\hline$T_{s 1}$ & $h(Z)$ & 0 & $\ldots$ & $1.4966 \mathrm{e}-01$ & $\ldots$ & $2.143 e+01$ & $\ldots$ & 0.837023 & $\ldots$ & $\ldots$ & $\ldots$ \\
\hline$C_{1}$ & $l(Z)$ & 0 & $\ldots$ & $-8.976 \mathrm{e}-03$ & $\ldots$ & $2.404 \mathrm{e}+01$ & $\ldots$ & -1.20034 & $\ldots$ & 6.54585 & $\ldots$ \\
\hline$T_{s 2}$ & $m(Z)$ & 0 & $\ldots$ & $2.824 \mathrm{e}+00$ & $\ldots$ & $3.125 \mathrm{e}+01$ & $\ldots$ & 0.251719 & $\ldots$ & 6.1953 & $\ldots$ \\
\hline$C_{2}$ & $m(Z)$ & 0 & $\ldots$ & $4.064 \mathrm{e}+00$ & $\ldots$ & $2.969 \mathrm{e}+01$ & $\ldots$ & 0.288187 & $\ldots$ & 8.3886 & $\ldots$ \\
\hline \multicolumn{12}{|c|}{ H-like $n=2$ Resonances } \\
\hline $\mathrm{N}$ & $f(I / Z)$ & 0 & $\ldots$ & $5.270 \mathrm{e}-03$ & $\ldots$ & $1.597 \mathrm{e}-02$ & $\ldots$ & 1.83289 & $\ldots$ & $\ldots$ & $\ldots$ \\
\hline$T_{s 1}$ & $k(Z)$ & 0 & $\ldots$ & $7.747 \mathrm{e}-03$ & $\ldots$ & $1.000 \mathrm{e}+01$ & $\ldots$ & -4.32176 & $\ldots$ & 6.47427 & $\ldots$ \\
\hline$C_{1}$ & $l(Z)$ & 0 & $\ldots$ & $2.797 e+00$ & $\ldots$ & $1.000 \mathrm{e}+01$ & $\ldots$ & 4.77743 & $\ldots$ & 2.20888 & $\ldots$ \\
\hline$T_{s 2}$ & $k(Z)$ & 0 & $\ldots$ & $1.627 \mathrm{e}-01$ & $\ldots$ & $1.000 \mathrm{e}+01$ & $\ldots$ & -1.28911 & $\ldots$ & 1.15294 & $\ldots$ \\
\hline$C_{2}$ & $k(Z)$ & 0 & $\ldots$ & $6.737 e+00$ & $\ldots$ & $1.000 \mathrm{e}+01$ & $\ldots$ & 0.426109 & $\ldots$ & -0.656385 & $\ldots$ \\
\hline
\end{tabular}




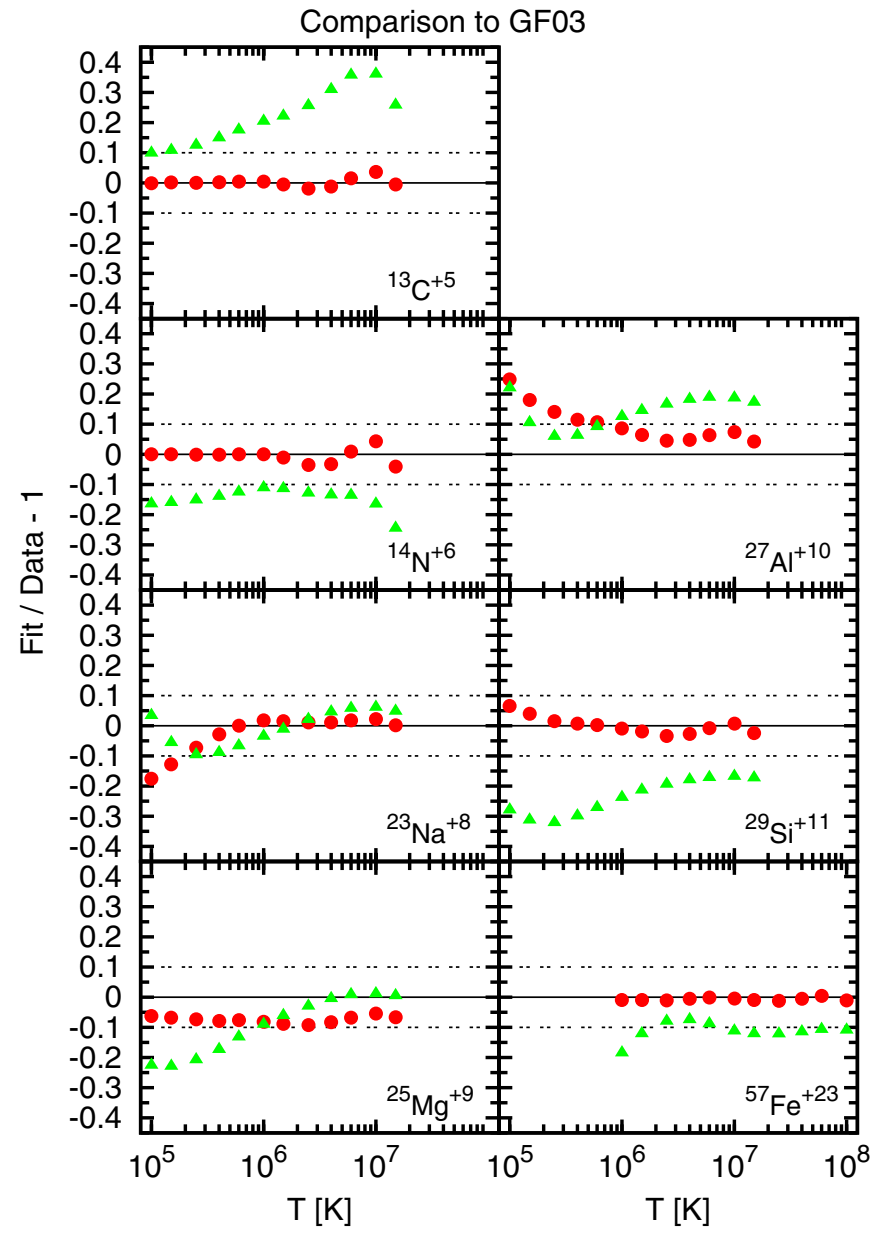

Figure 12. Comparison of the fit residuals to the total effective collision strength of the ZS00 data for the present work (red solid circles) and the work of GF03 (green solid triangles). The dashed lines indicate $\pm 10 \%$ deviations from the data.

(A color version of this figure is available in the online journal.)

employed in the present work results in at most a $10 \%$ deviation from the exact data. The exceptions are the Li-like sodium and aluminum ions, which possess $20 \%-30 \%$ deviations at the low end of the temperature range. Overall, the present approach results in more accurate fits than GF03, with the exception of the Li-like magnesium ion at temperatures greater than $10^{6} \mathrm{~K}$.

These fits have been implemented in CLOUDY as an update to the existing data of GF03. Note that in computing the collision strengths for the Syunyaev \& Churazov (1984) hydrogenic, and lithium-like ions, we have imposed an upper limit $\left(C_{1} \leqslant 1\right)$ on the power-law value for hydrogenic resonances, and limits on the power-law and temperature scaling values $\left(C_{1} \geqslant-1\right.$ and $\left.T_{s 1} \leqslant 35\right)$ for Li-like $n=3$ resonances, respectively, to prevent divergences to unphysical values. We adopt the lithium-like profiles for the sodium-like ions for lack of any numerical data. Such inadequacies illustrate the need for additional calculations primarily for hydrogenic and sodium-like ions.

\section{APPENDIX B}

\section{SED COMPILATION}

Because the hyperfine lines considered in this paper cover a wide spectral range (the shortest wavelength is $10 \mu \mathrm{m}$ ), and because indirect radiative excitations can be important, the spectral energy distribution (SED) over the entire electromagnetic spec-

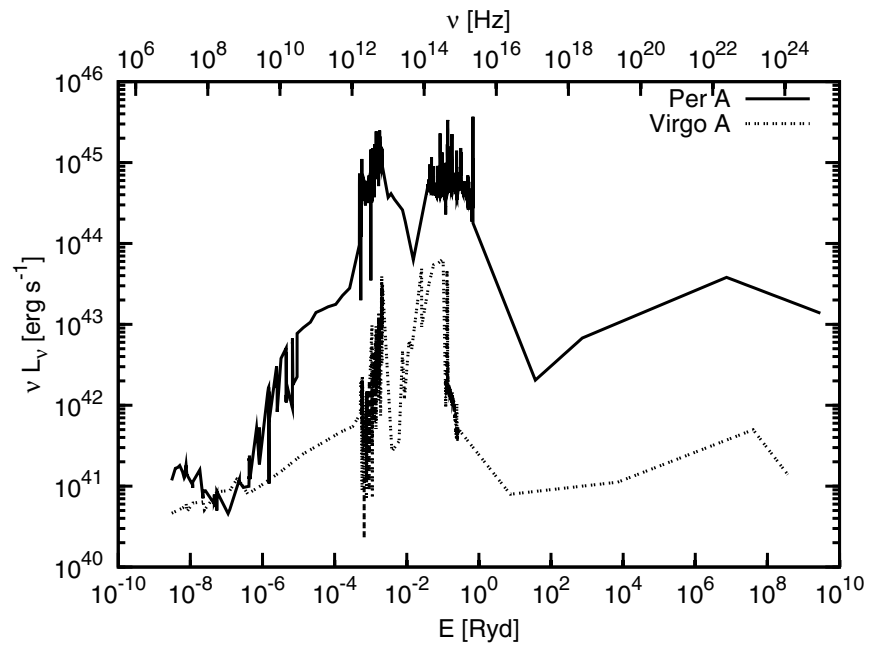

Figure 13. Spectral energy distribution of NGC 1275 and 3C 274. See the text for details.

trum of each source is required. In this Appendix, we discuss how we compiled the SEDs of NGC 1275 and 3C 274, shown in Figure 13. In both cases, we have used disjoint multiband observations mainly derived from the NASA/IPAC Extragalactic Database $\left(\mathrm{NED}^{4}\right)$.

In the case of NGC 1275, the spectral segments listed in Table 10 cover various epochs over the last 50 years, and are subject to the long term variability observed for this galaxy (Nesterov et al. 1995). This variability amounts to roughly a factor of 5 in the radio, and a factor of 2 in the optical, and it is thought to arise from accretion onto the central black hole, as well as jet interaction with the accreting gas. Currently, the AGNs are brightening, as seen, for example, in the millimeter observations of Trippe et al. (2011).

Obviously, arriving at an "average" SED requires accounting for temporal variability. The corrections are listed in Column 6 of Table 10. Because most observations were taken close to the light curve minimum, the temporal corrections are set to the square root of maximum variability. When long-term monitoring is absent, the optical factor is employed, that is, the correction is set to $\sqrt{2}$. The values for the millimeter and submillimeter variability are drawn from the $90 \mathrm{GHz}$ and $270 \mathrm{GHz}$ light curves of Nesterov et al. (1995).

Aperture corrections, shown in Column 7 of Table 10, are employed only for the near infrared, optical, and ultraviolet parts of the spectrum. The first two are obtained with long slit spectroscopy, while the latter with grism spectroscopy. The infrared and ultraviolet segments are adjusted to be roughly continuous with the optical. The joint spectrum is then corrected for reddening following the prescription of Fitzpatrick (1999) and adopting a $R_{V}=3.1$ extinction law, while its normalization is adjusted to yield a B-filter (Bessell 1990) magnitude in agreement with Moustakas \& Kennicutt (2006). No aperture corrections are employed with the other segments, either because the aperture contains the entire galaxy, or because it was not possible to estimate the total flux (e.g., MIR).

By contrast, the SED of 3C 274 refers to the nuclear galaxy region (roughly the central $35^{\prime \prime}$, or $3 \mathrm{kpc}$ ), due to the cluster's proximity to the Milky Way $(z=0.00436)$. In addition, no long term variability has been observed for this galaxy, and therefore no corrections are required.

\footnotetext{
4 http://ned.ipac.caltech.edu/
} 
Table 10

SED Compilation Details

\begin{tabular}{|c|c|c|c|c|c|c|c|}
\hline $\begin{array}{l}\text { Band } \\
\text { (1) }\end{array}$ & $\begin{array}{l}\text { Spectral Range } \\
\text { (2) }\end{array}$ & $\begin{array}{l}\text { Obs. Epoch } \\
\text { (3) }\end{array}$ & $\begin{array}{l}\text { Facility } \\
\text { (4) }\end{array}$ & $\begin{array}{l}\text { Aperture } \\
\text { (5) }\end{array}$ & $\begin{array}{l}V_{\text {corr }^{\mathrm{a}}} \\
(6)\end{array}$ & $\begin{array}{l}A_{\text {corr }}^{\mathrm{b}} \\
(7)\end{array}$ & $\begin{array}{l}\text { Ref. }^{c} \\
(8)\end{array}$ \\
\hline Radio-CM & $<30 \mathrm{GHz}$ & 1969-2007 & $\ldots$ & $\ldots$ & $\ldots$ & $\ldots$ & $\ldots$ \\
\hline Radio-MM & $30-857 \mathrm{GHz}$ & 2009 & Planck & $4^{\prime}-33^{\prime}$ & $\sqrt{5}$ & $\ldots$ & 1 \\
\hline FIR & 43-190 $\mu \mathrm{m}$ & 1998 & $I S O$ & $72^{\prime \prime}$ & $\sqrt{15}$ & $\ldots$ & 2 \\
\hline MIR & $12-100 \mu \mathrm{m}$ & 2006 & Bok & $75^{\prime \prime}$ & $\sqrt{2}$ & $\ldots$ & 3 \\
\hline MIR & 6-30 $\mu \mathrm{m}$ & 2005 & Spitzer & $11^{\prime \prime} \times 22^{\prime \prime}$ & $\sqrt{2}$ & $\ldots$ & 4 \\
\hline NIR & $0.8-2.4 \mu \mathrm{m}$ & 2003 & IRTF & $0^{\prime \prime} .8 \times 1^{\prime \prime} .5$ & $\sqrt{2}$ & 20 & 5 \\
\hline Optical & $0.37-0.69 \mu \mathrm{m}$ & 2006 & Bok & $2^{\prime \prime} \times 516^{\prime \prime}$ & $\sqrt{2}$ & 20 & 6 \\
\hline UV & $0.13-0.28 \mu \mathrm{m}$ & 2005 & GALEX & $10^{\prime \prime}$ & $\sqrt{2}$ & 10 & 7 \\
\hline X-ray soft & $0.3-100 \mathrm{keV}$ & 2005-2007 & XMM-Newton & $25^{\prime \prime}$ & $\sqrt{2}$ & $\ldots$ & 8 \\
\hline X-ray hard & $0.3-100 \mathrm{keV}$ & 2005-2007 & Swift & $17^{\prime}$ & $\sqrt{2}$ & $\ldots$ & 8 \\
\hline$\gamma$-ray & $0.2-10 \mathrm{GeV}$ & 2008 & Fermi & $2^{\circ}-0.1^{\circ}$ & $\sqrt{2}$ & $\ldots$ & 9 \\
\hline
\end{tabular}

Notes.

${ }^{a}$ Variability correction factor.

${ }^{\mathrm{b}}$ Aperture correction factor.

${ }^{c}$ Reference List: (1) León-Tavares et al. (2012); (2) Brauher et al. (2008); (3) Moustakas \& Kennicutt (2006); (4) Weedman et al. (2005);

(5) Riffel et al. (2006); (6) Buttiglione et al. (2009); (7) GALEX coadded spectral products (http://galex.stsci.edu/GR6/); (8) Ajello et al. (2010);

(9) Abdo et al. (2009).

\section{REFERENCES}

Abdo, A. A., Ackermann, M., Ajello, M., et al. 2009, ApJ, 699, 31

Ajello, M., Rebusco, P., Cappelluti, N., et al. 2010, ApJ, 725, 1688

Asplund, M., Grevesse, N., Sauval, A. J., \& Scott, P. 2009, ARA\&A, 47,481

Bania, T. M., Balser, D. S., Rood, R. T., Wilson, T. L., \& LaRocque, J. M. 2007, ApJ, 664, 915

Bania, T. M., Balser, D. S., Rood, R. T., Wilson, T. L., \& Wilson, T. J. 1997, ApJS, 113,353

Bessell, M. S. 1990, PASP, 102, 1181

Brauher, J. R., Dale, D. A., \& Helou, G. 2008, ApJS, 178, 280

Buttiglione, S., Capetti, A., Celotti, A., et al. 2009, A\&A, 495, 1033

Chatzikos, M., Ferland, G. J., \& Williams, R. J. R. 2013, ApJ, 779, 122

Churazov, E., Forman, W., Jones, C., Sunyaev, R., \& Böhringer, H. 2004, MNRAS, 347, 29

Churazov, E., Forman, W., Vikhlinin, A., et al. 2008, MNRAS, 388, 1062

D’Cruz, N. L., Sarazin, C. L., \& Dubau, J. 1998, ApJ, 501, 414 (DSD98)

Dere, K. P., Landi, E., Mason, H. E., Monsignori Fossi, B. C., \& Young, P. R. 1997, A\&AS, 125, 149

Dickey, J. M., \& Lockman, F. J. 1990, ARA\&A, 28, 215

Diehl, R. 2002, NewAR, 46, 547

Ettori, S., Fabian, A. C., \& White, D. A. 1998, MNRAS, 300, 837

Fabian, A. C., Sanders, J. S., Taylor, G. B., et al. 2006, MNRAS, 366, 417

Ferland, G. J., Porter, R. L., van Hoof, P. A. M., et al. 2013, RMxAA, 49, 137

Fitzpatrick, E. L. 1999, PASP, 111, 63

Forman, W., Jones, C., Churazov, E., et al. 2007, ApJ, 665, 1057

Garstang, R. H. 1995, ApJ, 447, 962

Goddard, W. E., \& Ferland, G. J. 2003, PASP, 115, 647

Gould, R. J. 1994, ApJ, 423, 522

Grevesse, N., Asplund, M., Sauval, A. J., \& Scott, P. 2010, Ap\&SS, 328,179

Güdel, M., Briggs, K. R., Montmerle, T., et al. 2008, Sci, 319, 309
Guo, Z., Burrows, D. N., Sanders, W. T., Snowden, S. L., \& Penprase, B. E. 1995, ApJ, 453, 256

Landi, E., Young, P. R., Dere, K. P., Del Zanna, G., \& Mason, H. E. 2013, ApJ, 763,86

León-Tavares, J., Valtaoja, E., Giommi, P., et al. 2012, ApJ, 754, 23

Liang, H., Dickey, J. M., Moorey, G., \& Ekers, R. D. 1997, A\&A, 326, 108

Meynet, G., Arnould, M., Paulus, G., \& Maeder, A. 2001, SSRv, 99, 73

Million, E. T., Werner, N., Simionescu, A., \& Allen, S. W. 2011, MNRAS, 418,2744

Moustakas, J., \& Kennicutt, R. C., Jr. 2006, ApJS, 164, 81

Nesterov, N. S., Lyuty, V. M., \& Valtaoja, E. 1995, A\&A, 296, 628

Peterson, J. R., \& Fabian, A. C. 2006, PhR, 427, 1

Pettini, M., \& Bowen, D. V. 2001, ApJ, 560, 41

Reid, M. J., Menten, K. M., Zheng, X. W., et al. 2009, ApJ, 700, 137

Riffel, R., Rodríguez-Ardila, A., \& Pastoriza, M. G. 2006, A\&A, 457, 61

Rogers, A. E. E., Dudevoir, K. A., \& Bania, T. M. 2007, AJ, 133, 1625

Rood, R. T., Bania, T. M., \& Balser, D. S. 2007, in An Old Dog's Last Hunt: The Last Observations of the NRAO Green Bank 140 Foot Radio Telescope, ed. F. J. Lockman, F. D. Ghigo, \& D. S. Balser (The National Radio Astronomy Observatory), 451

Seaton, M. J. 2005, MNRAS, 362, L1

Shabaev, V. M., Shabaeva, M. B., \& Tupitsyn, I. I. 1995, PhRvA, 52, 3686

Stone, N. J. 2005, ADNDT, 90, 75

Sunyaev, R. A., \& Docenko, D. O. 2007, AstL, 33, 67

Sunyaev, R. A., Norman, M. L., \& Bryan, G. L. 2003, AstL, 29, 783

Syunyaev, R. A., \& Churazov, E. M. 1984, SvAL, 10, 201

Trippe, S., Krips, M., Piétu, V., et al. 2011, A\&A, 533, A97

Tully, R. B., \& Fisher, J. R. 1977, A\&A, 54, 661

Weedman, D. W., Hao, L., Higdon, S. J. U., et al. 2005, ApJ, 633, 706

Werner, N., Simionescu, A., Million, E. T., et al. 2010, MNRAS, 407, 2063

Zhang, H. L., \& Sampson, D. H. 2000, PhRvA, 61, 022707 (ZS00)

Zhang, H. L., \& Sampson, D. H. 2001, MNRAS, 322, 433 (ZS00) 